\title{
Optical MEMS for Lightwave Communication
}

\author{
Ming C. Wu, Fellow, IEEE, Olav Solgaard, Member, IEEE, and Joseph E. Ford
}

Invited Paper

\begin{abstract}
The intensive investment in optical microelectromechanical systems (MEMS) in the last decade has led to many successful components that satisfy the requirements of lightwave communication networks. In this paper, we review the current state of the art of MEMS devices and subsystems for lightwave communication applications. Depending on the design, these components can either be broadband (wavelength independent) or wavelength selective. Broadband devices include optical switches, crossconnects, optical attenuators, and data modulators, while wavelength-selective components encompass wavelength add/drop multiplexers, wavelength-selective switches and crossconnects, spectral equalizers, dispersion compensators, spectrometers, and tunable lasers. Integration of MEMS and planar lightwave circuits, microresonators, and photonic crystals could lead to further reduction in size and cost.
\end{abstract}

Index Terms-Microelectromechanical devices, optical fiber communication, optical signal processing, optical switches.

\section{INTRODUCTION}

$\mathbf{N}$ EARLY three decades ago, Petersen published a paper on the micromechanical spatial light modulator (SLM) array [1] and another on the silicon torsion mirror [2]. Thirty years later, this has become a thriving field known as optical microelectromechanical systems (MEMS), sometimes also called microoptoelectromechanical systems, with several conferences dedicated to the field. It is a key enabling technology for the "dynamic" processing of optical signals. The first market driver of optical MEMS was display [3], [4]. The digital micromirror devices developed by Texas Instruments Incorporated are one of the most successful MEMS products. They are now widely used in portable projectors, large-screen TVs, and digital cinemas [3]. The applications of optical MEMS in telecommunications started in the 1990s [5], [6]. Early efforts

Manuscript received July 7, 2006; revised October 2, 2006. This work was supported in part by the U.S. Defense Advanced Research Project Agency (DARPA)/Army Research Office under Grant W911NF-05-1-0359 and DARPA under Grant MDA972-02-1-0020.

M. C. Wu is with the Berkeley Sensor and Actuator Center (BSAC) and Electrical Engineering and Computer Sciences Department, University of California, Berkeley, CA 94720 USA (e-mail: wu@ eecs.berkeley.edu).

O. Solgaard is with the E. L. Ginzton Laboratory, Stanford University, Stanford, CA 94305 USA (e-mail: solgaard@ stanford.edu).

J. E. Ford is with the Department of Electrical and Computer Engineering, University of California, San Diego, CA 92093-0407 USA (e-mail: jeford@ ucsd.edu).

Color versions of Figs. 3, 5, 10-12, 14, 15, 17, 18, 20, 22, and 25-28 are available online at http://ieeexplore.ieee.org.

Digital Object Identifier 10.1109/JLT.2006.886405 have focused on the development of optical MEMS devices and fabrication technologies [7]-[10]. The telecom boom in the late 1990s and early 2000s has accelerated maturation of the technology. A wide range of optical MEMS components were taken from laboratories to reliable products that meet Telcordia qualifications. Although not all commercialization endeavors were successful due to the market downturn, the technology developed is available for new applications in communications and other areas [11].

In this paper, we will review the recent developments in optical MEMS for communication applications. With the rapid expansion of the field and proliferation of literature, it is not possible to cover all developments in the last decade. Instead, we will focus on a selected set of applications and discuss the design tradeoffs in MEMS devices and systems. Topics selected in this paper include optical switches, filters, dispersion compensators, spectral equalizers, spectrometers, tunable lasers, and other dense-wavelength-division-multiplexing (DWDM) devices such as wavelength add/drop multiplexers (WADMs), wavelength-selective switches (WSSs), and wavelengthselective crossconnects (WSXC). Most of the practical components reported were based on free-space optics. There are increasing interests in extending the benefits of optical MEMS to guided-wave optics or even nanoscopic photonic structures. This new trend will be discussed at the end of this paper.

Various types of optical switches are needed in telecommunication networks. Small $1 \times N$ and $N \times N$ switches are useful for protection, while optical crossconnect (OXC) offers fast provisioning and network management at the wavelength level. Nodes in ring networks employ WADMs. As the networks evolve toward mesh configuration, WSSs and WSXC become important. Dispersion compensators and spectral equalizers are essential for improving the link performance as the data rates approach $40 \mathrm{~Gb} / \mathrm{s}$. Spectral filters and tunable lasers increase the flexibility of DWDM nodes.

This paper is organized as follows: Section II discusses broadband (wavelength-independent) devices, including data modulators, variable optical attenuators (VOAs), and twodimensional (2-D) and three-dimensional (3-D) MEMS optical switches. Section III describes wavelength-selective MEMS, including spectral equalizers, WADMs, WSSs, WSXCs, filters, dispersion compensators, transform spectrometers, and tunable lasers. Section IV focuses on the integration of MEMS and planar lightwave circuits (PLC). Section V introduces new device concepts based on MEMS-actuated microresonators and photonic crystals, and Section VI concludes this paper. 


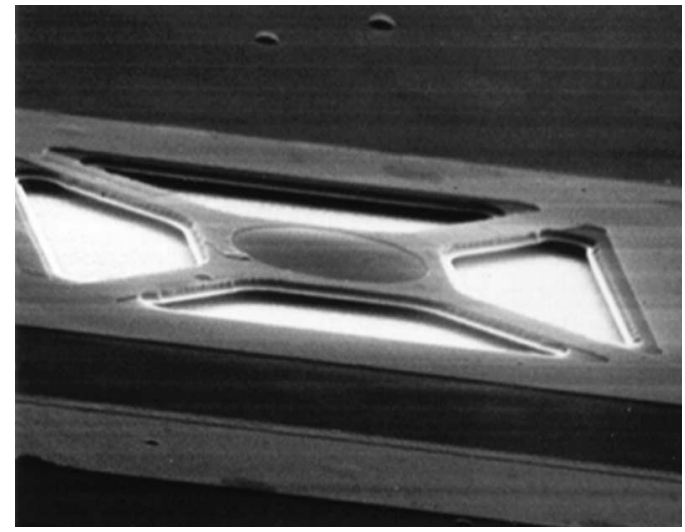

Fig. 1. MEMS etalon modulator used for digital data modulation at over $1 \mathrm{Mb} / \mathrm{s}$. The circular optical aperture is $22 \mu \mathrm{m}$ in diameter.

\section{WAVELENGTH-INDEPENDENT MEMS}

\section{A. Data Modulators}

The first practical application of MEMS devices in fiber communications was as an optical data modulator, originally intended for a low-cost fiber-to-the-home network. A modulator is essentially a $1 \times 1$ switch, operated in either transmission (two fibers) or reflection (single fiber). The optical power is provided by a constant-intensity remote source, and the modulator imprints a data signal by opening and closing in response to an applied voltage. Signaling in DWDM fiber networks usually requires an expensive wavelength-controlled laser at each remote terminal. Passive data modulators offered a potentially inexpensive solution, but waveguide modulators were too expensive and too narrow in optical spectral bandwidth to be practical. MEMS offered a new and practical solution.

The mechanical antireflection switch (MARS) modulator is a variable air-gap etalon operated in reflection. The basic structure is a quarter-wave dielectric antireflection (AR) coating suspended above a silicon substrate [5]. The quarter-wave layer is made of silicon nitride with $1 / 4 \lambda$ optical path (index times thickness), which is roughly $0.2 \mu \mathrm{m}$ for the $1550-\mathrm{nm}$ telecom wavelength. The mechanically active silicon nitride layer is suspended over an air gap created by a $3 / 4 \lambda$-thick phosphosilicate glass sacrificial layer $(0.6 \mu \mathrm{m})$. Without deformation, the device acts as a dielectric mirror with about $70 \%(-1.5-\mathrm{dB})$ reflectivity. Voltage applied to electrodes on top of the membrane creates an electrostatic force and pulls the membrane closer to the substrate, while membrane tension provides a linear restoring force. When the membrane gap is reduced to $\lambda / 2$, the layer becomes an AR coating with close to zero reflectivity. A switching contrast ratio of $10 \mathrm{~dB}$ or more was readily achieved over a wide (30-nm) spectral bandwidth.

The initial MARS device shown in Fig. 1 consisted of a $22-\mu \mathrm{m}$ optical window supported by $\mathrm{X}$-shaped arms and had a resonant frequency of 1.1 MHz. Later devices used a higheryield structure with a symmetric "drum head" geometry [12], [13]. These devices were capable of relatively high-speed operation: by optimizing the size and spacing of the etch, access holes provide critical mechanical damping, and digital modulation above $16 \mathrm{Mb} / \mathrm{s}$ was demonstrated [14]. While such data rates are no longer relevant for telecom, even for fiber-to-

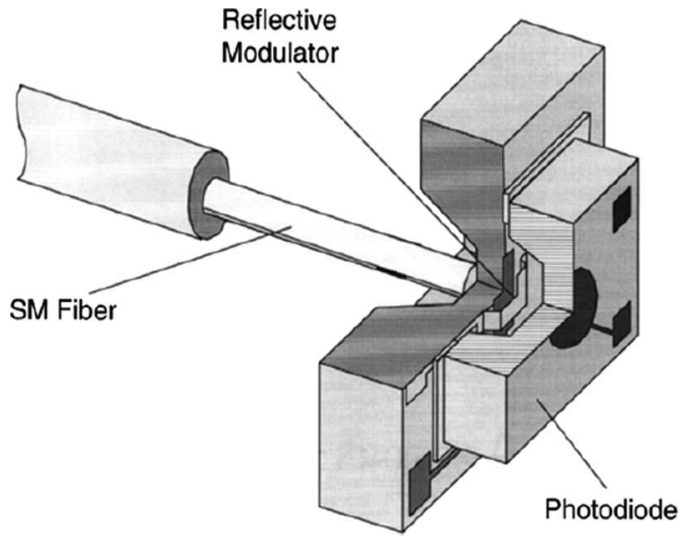

Fig. 2. Package configuration for a MEMS data transceiver.

the-home, related modulators are useful for low-power dissipation telemetry from remote sensors using free-space optical communications.

These early devices provided a proving ground for the reliability and packaging of optical MEMS telecom components. Initial skepticism from conservative telecom engineers was combated by the parallel testing of device array operated for months to provide trillions of operating cycles. The packaging of optical MEMS devices provided new challenges for MEMS engineers, but the simple end-coupled configuration was relatively straightforward to implement. Fig. 2 shows the configuration for a duplex modulator incorporating a MEMS etalon, where data can be received by a photodiode and transmitted by modulating the etalon reflectivity [15].

\section{B. Variable Attenuators}

Data modulators are operated with digital signals, but the fundamental response of an etalon modulator is analog. Electrically controlled VOAs at that time were constructed with bulk optical components with electromechanical actuation, with 10-100-ms response. Erbium fiber amplifiers can use VOA to suppress transient power surges, but the time scale required was $10 \mu \mathrm{s}$, much slower than the data modulation rate. MEMS provided an attractive replacement for optomechanical VOAs, and this turned out to be the first volume application for MEMS devices in telecom networks.

The first MEMS VOA was fabricated by scaling the optical aperture of a MARS modulator from 25 to $300 \mu \mathrm{m}$ so that it could be illuminated with a collimated beam. The reflected signal was focused into a separate output fiber, avoiding the need for external splitters or circulators to separate the output signal [16]. The first such VOA device is shown in Fig. 3. The wavelength dependence of a simple etalon was reduced using a more complex three-layer dielectric stack as the mechanically active structure, where the original $1 / 4 \lambda$ silicon nitride layer is sandwiched between conductive polysilicon top $(1 / 2 \lambda$ thickness) and bottom ( $1 / 4 \lambda$ thickness) layers. This attenuator provided fast $(3 \mu \mathrm{s})$ response with $30-\mathrm{dB}$ controllable attenuation over the 40-nm operating bandwidth, with 0.06-dB polarization-dependent loss, and also supported the 100-mW power level present in amplifiers. However, the 3-dB insertion loss was excessive. 


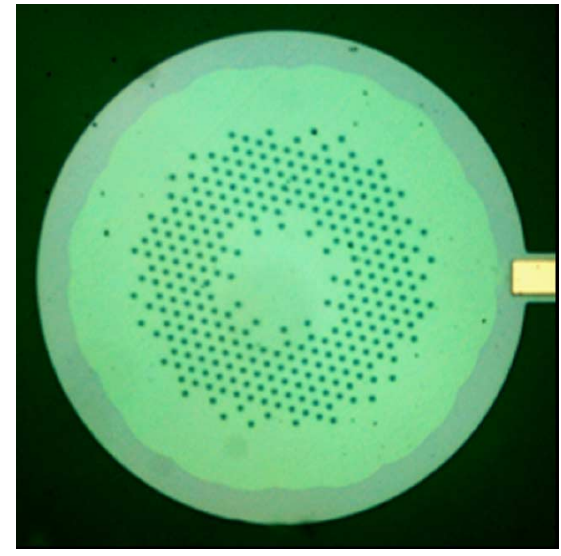

Fig. 3. MEMS etalon variable attenuator using a 0.5-mm diameter drumhead geometry. The lighter area covers an air gap between the silicon substrate. The hexagonally distributed spots are etch access holes.

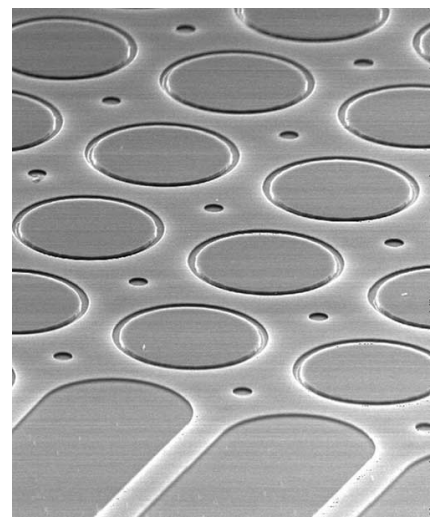

Fig. 4. Lightconnect's diffractive MEMS VOA.

The most direct possible approach to attenuation is to use a MEMS actuator to insert an optical block between the input and output fiber. This was implemented with a surface micromachining (MUMPS process) [17] and with a comb-driven silicon-on-insulator (SOI) device [18]. Such VOAs offered excellent dynamic range (measurement limited at $90 \mathrm{~dB}$ ), but the polarization-dependent loss could be large $(\gg 1 \mathrm{~dB})$ at high attenuations.

Further improvement was needed and was made. Combining the collimated beam geometry with a first-surface torsion mirror reflector provided a low-insertion-loss structure with excellent spectral and polarization performance. For example, the device demonstrated by Isamoto et al. [19] achieved 40-dB attenuation with a $600-\mu \mathrm{m}$ mirror driven with $5 \mathrm{~V}$ to tilt up to $0.3^{\circ}$. Similar configurations were commercialized, although the specific designs have not been published.

Another commercial MEMS VOA is based on a diffractive MEMS device [4] also used with a collimated beam. This device provides excellent optical performance as well as high speed: stable operation with $30-\mathrm{dB}$ contrast and less than $40-\mu$ s response time using an $8-\mathrm{V}$ drive. A novel structure with circularly symmetric features, shown in Fig. 4, was used to suppress the polarization-dependent loss to under $0.2 \mathrm{~dB}$ [20]. This device was one of the first Telcordia-qualified MEMS components, with 40000 units reportedly shipped by 2005 [21].

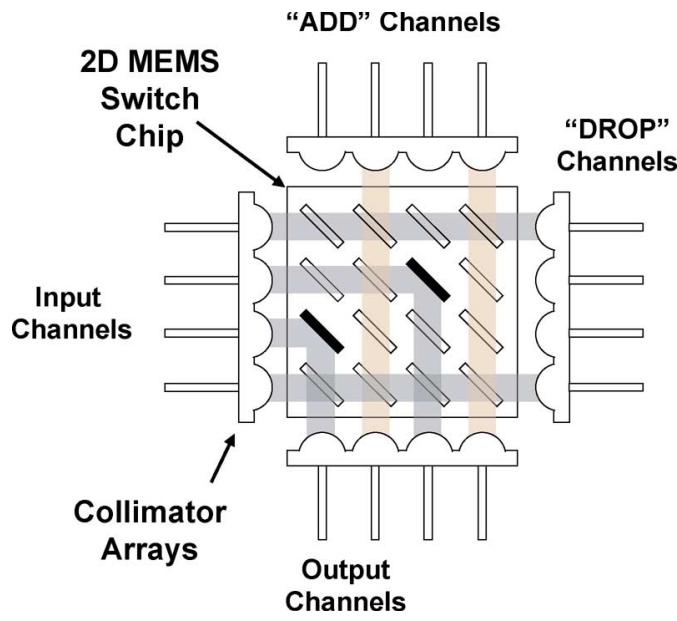

Fig. 5. Schematic of 2-D MEMS optical switches.

\section{Two-Dimensional MEMS Switches}

Protection switches are made of $1 \times N$ or small $N \times N$ switches. This can be realized by a 2-D array of vertical micromirrors commonly known as a 2-D MEMS switch. Fig. 5 shows the generic schematic of such a switch. The optical beams are collimated to reduce diffraction loss. The micromirrors are "digital": They either direct the optical beams to the orthogonal output ports or pass them to the drop ports. Generally, only one micromirror in a column or row is in the reflection position during operation.

The first MEMS 2-D switch $(2 \times 2)$ was reported in [22] and quickly followed by related work [23], [24]. For $2 \times 2$ switches, low insertion loss $(0.6 \mathrm{~dB})$ can be achieved without using collimators, especially when the micromirror is immersed in index-matching fluid [25]. Latchable $2 \times 2$ switches incorporating MEMS bistable structures were later commercialized [26], [27]. Larger switches require optical collimators to reduce diffraction loss. Switches with $8 \times 8$ and $16 \times 16$ ports were demonstrated [28], [29]. There are two basic approaches for the actuation of the micromirror. The first is based on the rotation of the micromirror [22], [28], [30], [31]. The mirror is initially parallel to the substrate (OFF position). When actuated, it is rotated to the vertical position (ON). The second approach moves the vertical micromirrors in and out of the optical paths without changing the mirror angle [23]-[25], [29], [32], [33]. The 2-D switches have been realized by both bulk-micromachining [22]-[25] and surface-micromachining [28]-[30], [32] technologies. Electrostatic actuation is most commonly used [22]-[29], [32]. Magnetic actuation has also been demonstrated [23], with some in conjunction with electrostatic clamping [30].

The port count of 2-D switches is determined by several factors, including mirror angle, size, fill factor (mirror width divided by unit cell width), and curvature. The expandability of the 2-D switch has been studied in [34] and [35]. To minimize optical diffraction loss, a confocal geometry is used with the average optical path length equal to the Rayleigh range, which is proportional to the square of the optical beam waist. Larger mirrors are therefore required to support longer Rayleigh length in higher port-count switches. In an $N \times N$ switch, the mirror size scales as $N$, whereas 


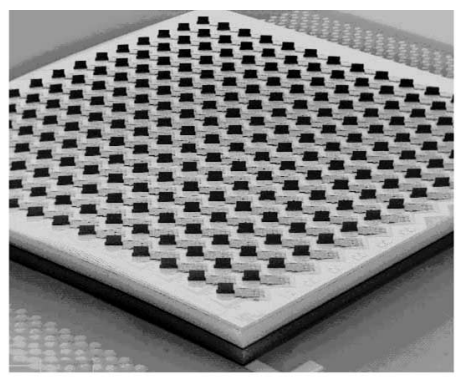

(a)

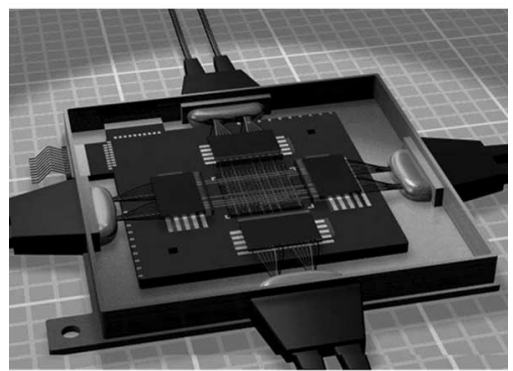

(b)

Fig. 6. (a) SEM of OMM's $16 \times 16$ switch (reprinted from [29] with permission). (b) Photograph of the packaged switch (reprinted from [36] with permission).

the linear dimension of the chip scales as $N^{2}$ [35]. Large chips are more susceptible to imperfections in mirror angles, which cause walkoff of optical beams at the receiving fibers. Ultimately, the chip size will be limited by the fabrication precision of the micromirrors. $16 \times 16$ switches have been realized, and $32 \times 32$ switches are within the capability of today's technology.

Fig. 6(a) shows a scanning electron micrograph (SEM) of OMM's 2-D switch [29]. A vertical mirror is attached at the tip of a cantilever. The tilted cantilever can be pulled down electrostatically. The mirror angle is maintained at $90^{\circ}$ during switching. The switch is fabricated using a standard threepolysilicon-layer surface-micromachining process. The mirrors are assembled into vertical position with angular distribution of $(90 \pm 0.1)^{\circ}$. The hermetic switch package is shown in Fig. 6(b) [36]. Maximum insertion losses of 1.7 and $3.1 \mathrm{~dB}$ have been obtained for $8 \times 8$ and $16 \times 16$ switches, respectively, and the crosstalk is less than $-50 \mathrm{~dB}$. The switching time is less than $7 \mathrm{~ms}$. Packaging is critical to attain long-term reliability and satisfy Telcordia qualification for telecommunication applications [36].

There were also significant efforts in nonmirror-based MEMS 2-D switches [37], [38]. Both Agilent's Champaign switch [37] and NTT's OLIVE switch [38] used microfluidic actuation to switch light between intersecting waveguides. The Champaign switch used thermally generated bubbles to displace index-matching fluids at waveguide intersections, causing the light to bend by total internal reflection (TIR). The OLIVE switch used thermal-capillary force to move trapped bubbles. One drawback of these approaches is the cumulative losses and crosstalks through multiple waveguide intersections. The maximum port counts achieved are $32 \times 32$ and $16 \times 16$ for the Champaign and the OLIVE switches, respectively.

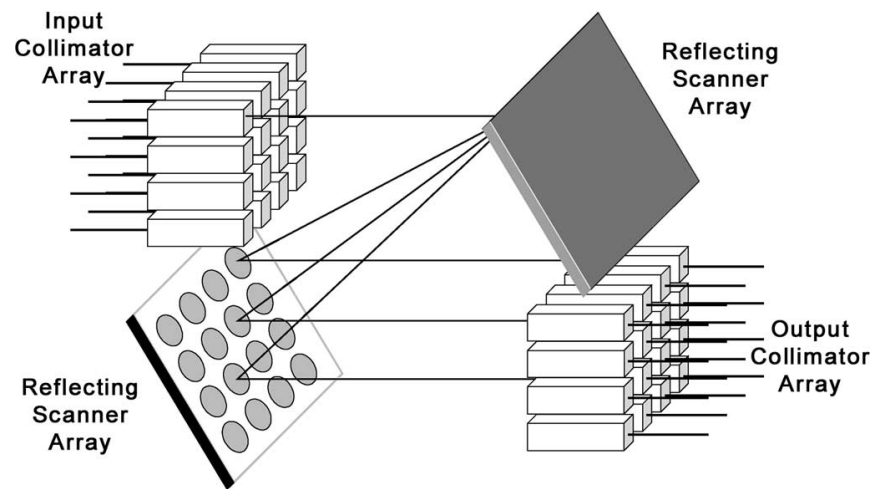

Fig. 7. Schematic of a 3-D MEMS switch.

\section{Three-Dimensional MEMS Switches}

A transparent optical crossconnect (OXC) with large port count can be realized by 3-D MEMS switches illustrated in Fig. 7. The input and output fibers are arranged in 2-D arrays. The optical beams are steered in three dimensions by two stages of dual-axis micromirrors, directing it toward the desired output port. The 3-D MEMS switch has a favorable scaling law with respect to port count: Assuming the maximum scan angle of the mirror is fixed, the optical path length is proportional to $N$ in an $N \times N$ switch. To maintain confocal configuration for minimum loss, the beam waist, and therefore the mirror size, needs to scale as $\sqrt{N}$. As a result, the linear dimension of the mirror chip scales as $\sqrt{N} \cdot \sqrt{N}=N$ [39]-[41]. In addition, it has low and uniform insertion loss. The 3-D MEMS OXC is a subject of intense interest during the telecom boom around the turn of the century [42]-[46]. Early efforts (before 2002) focused on OXCs with port count $\sim 1000 \times 1000$ [47], [48], driven by the explosion of Internet data transport. Recently, interest has shifted to applications in metropolitan area networks, including metro access and metro core networks, which requires OXC with medium port count $(\sim 100 \times 100)$, with emphasis on low cost, low-power consumption, and small footprint [44], [49]. Our discussion here will focus on this trend.

Detailed design tradeoffs and system implementations of the 3-D MEMS OXC have been reported recently [42]-[46]. Two schemes have been proposed to reduce the size of the switch and tilt angle of the micromirror. Lucent inserted a Fourier lens between the two micromirror chips with the focal length equal to the Rayleigh range of the optical beam (Fig. 8) [50]. This reduces the required scan angle of the mirror. In addition, the mirrors can be placed at the beam waist, resulting in $\sqrt{2}$ times smaller optical beams. This permits the use of smaller mirrors and/or reduction of the crosstalk. Fujitsu used a "rooftop" mirror to connect two adjacent micromirror chips (photograph show in Fig. 9) [44]. The rooftop mirror shifts the optical beams laterally, reducing the tilt angle requirement. Folding of the optical beam also shrinks the footprint of the switch.

In the compact switch category, Lucent's $64 \times 64$ switch has a size of $100 \times 120 \times 20 \mathrm{~mm}^{3}$, which can be mounted on a standard circuit board [49]. The insertion loss is $1.9 \mathrm{~dB}$. Fujitsu's $80 \times 80$ switch has a packaged size of $77 \times 87 \times$ $53 \mathrm{~mm}^{3}$ [44]. The average insertion loss is $2.6 \mathrm{~dB}$. Impressively, the switch continues to operate under vibration or 50G shock 


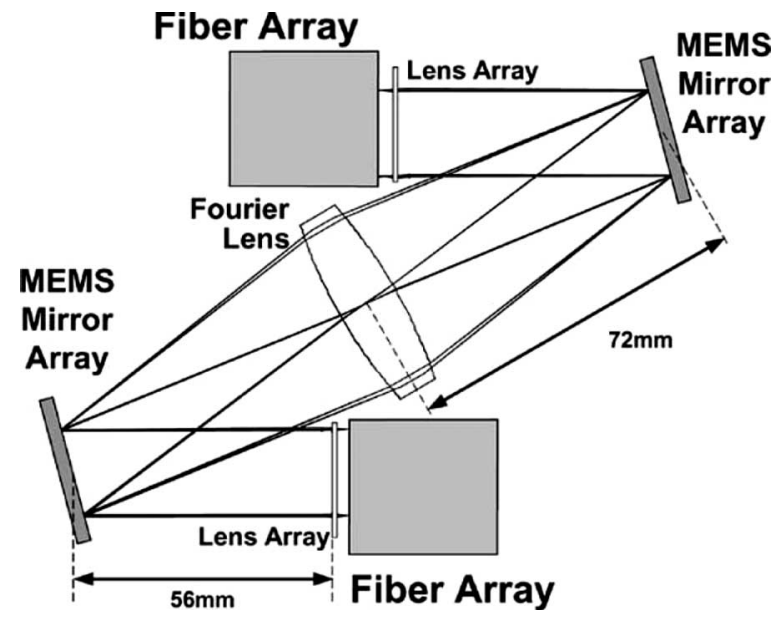

Fig. 8. Lucent's optical system layout for OXC (reprinted from [50] with permission). A Fourier lens is inserted between the two MEMS chips to reduce the required tilt of the mirror and beam size.

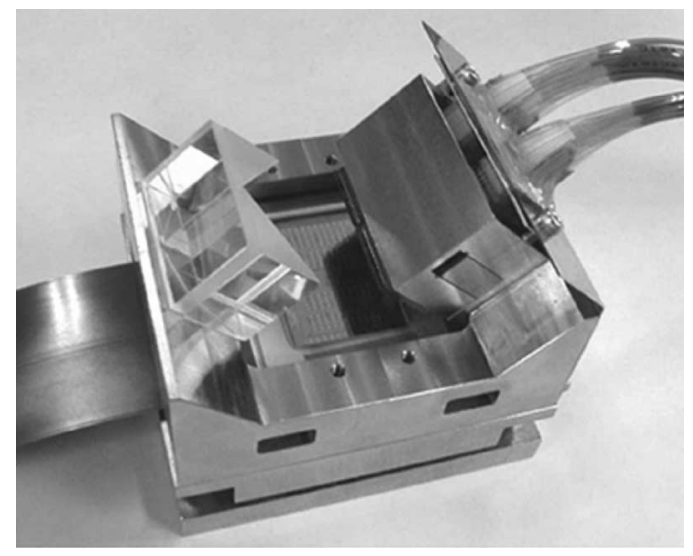

Fig. 9. Photograph of Fujitsu's $80 \times 80$ OXC with a rooftop reflector connecting the two MEMS chips (reprinted from [44] with permission). The packaged size is $77 \times 87 \times 53 \mathrm{~mm}^{3}$.

without any signal degradation. The total power consumption of Fujitsu's switch is only $8.5 \mathrm{~W}$, thanks to the low operating voltage of the mirrors. NTT's $100 \times 100$ switch has a size of $80 \times 60 \times 35 \mathrm{~mm}^{3}$ with an insertion loss of $4 \mathrm{~dB}$ [43].

The two-axis micromirror array is the key enabling device of the 3-D switch. Important parameters include size, tilt angle, flatness, fill factor, and resonant frequency of the mirror. Additionally, the stability of the mirror plays a critical role in the complexity of the control schemes. Early development focused on surface-micromachined two-axis scanners [51], [52]. The residue stress limits the mirror size to approximately $1 \mathrm{~mm}$, and the different thermal expansion coefficients between the mirror and the metal coating also cause the mirror curvature to change with temperature. Bulk-micromachined single-crystalline silicon micromirrors are often used in highport-count OXCs that require larger mirror size [46], [53]-[56].

Electrostatic actuation is most commonly used because of its low-power consumption and ease of control. Early devices use parallel-plate actuators, which have high actuation voltage and limited scan angle due to pull-in instability [57]. Although the pull-in effect can be mitigated by nonlinear controllers, it

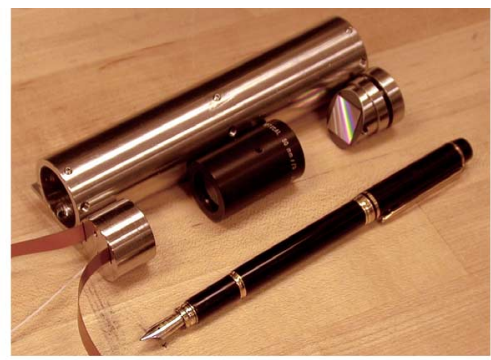

(a)
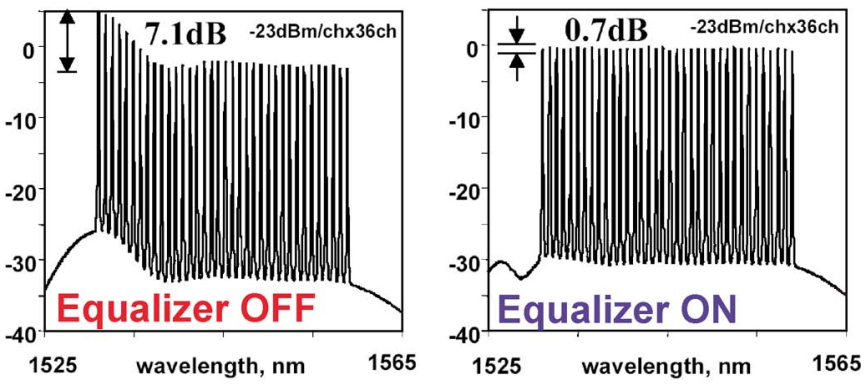

(b)

Fig. 10. (a) Dynamic spectral equalizer package and (b) transmission spectra showing the improvement in channel uniformity for a 36-channel DWDM transmission.

increases the complexity of electronics [58]. Micromirrors with vertical comb drive actuators, first reported in [59], offer many advantages. They have a much larger torque, which one can use to reduce the operating voltage as well as increase the resonant frequency. In addition, they are free from the pull-in effect, further increasing the stable tilt angles. It should be mentioned that lateral pull-in between comb fingers is a potential issue but could be mitigated by MEMS design (such as V-shaped torsion beam [60] or off-centered combs [61]). Several variations of vertical comb drive mirrors have been reported, including selfaligned vertical combs [62], [63], angular vertical combs [64], [65], electrostatically assembled vertical combs [66], and thick vertical combs $(100 \mu \mathrm{m})$ attached to mirror edges on doublesided SOI wafers [44], [60].

\section{W AVELENGTH-SELECTIVE MEMS}

\section{A. Spectral Equalizers}

The natural extension of a single variable attenuator is to provide a VOA for each channel of a DWDM transmission system. The surface-normal geometries of the etalon mirrorand grating-based attenuators discussed in Section II-B were all compatible with a free-space imaging spectrometer. An input fiber is imaged through a diffraction grating so that each spectral channel is laterally shifted to illuminate one modulator in a linear array. The reflected signal, attenuated to the desired value, is collected into a single output fiber by a second pass through the imaging spectrometer. The first such MEMS spectral equalizer used a continuous etalon membrane [67]. This approach was later implemented in the compact package shown in Fig. 10, which located the MEMS device array next to a single input/output (I/O) fiber. A single lens is to collimate the multiwavelength beam onto a blazed reflective grating and 


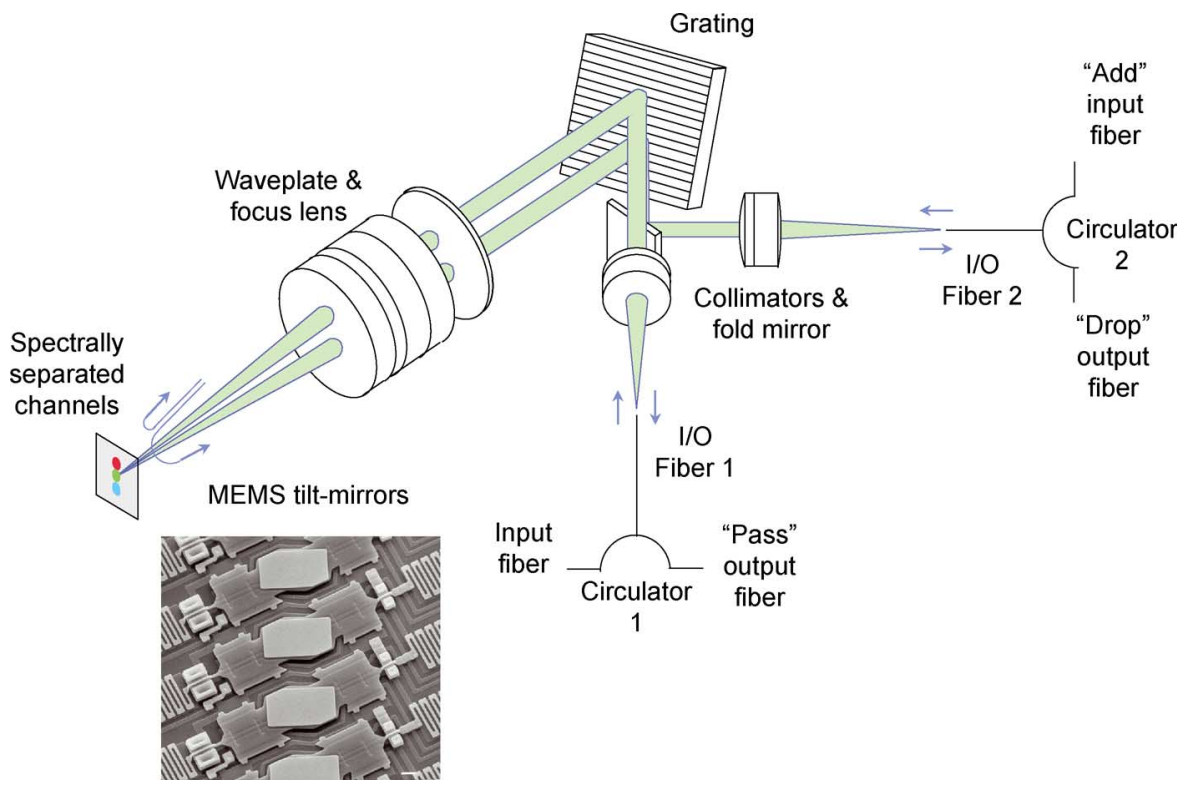

Fig. 11. Optical schematic for a $2 \times 2$ MEMS wavelength add/drop switch.

refocus the spectrally separated signals with a second pass onto the MEMS array. A third and fourth pass through the lens reintegrates the signal into the I/O fiber, where it is separated by an external optical circulator. The use of such equalizers is illustrated by the before and after spectral traces at the bottom of Fig. 10, showing the improvement in uniformity of 36 channels sent through a two-stage amplifier. The equalizer setting was generated by an iterative algorithm running on the computer controller [68].

Dynamic spectral equalization quickly went from an option to a practical requirement as the channel transmission rate increased from 2.5 to 10 and then to $40 \mathrm{~Gb} / \mathrm{s}$. The simplest and least expensive dynamic gain equalizers (DGEs) use a mid-amplifier filter that can be spectrally uniform (a VOA, as discussed above) or provide a constant spectral slope [69]. Two distinct categories of spectral equalizers emerged. DGEs provide a smoothly varying spectral profile used to compensate for the varying gain profiles in amplifiers, while dynamic channel equalizers (DCEs) provide the discrete channel-by-channel power adjustment needed to compensate for nonuniform transmission source intensity or path-dependent loss. Channel equalizers are preferable in general but require accurate matching of the equalizer passband to the transmission grid to avoid passband narrowing.

Channel equalizers were implemented using discrete VOAs attached to waveguide spectral multiplexers [70] and using an oversampled array of digital tilt mirrors [71]. However, the best performance in channel equalizers was achieved by combining the type of free-space grating demultiplexer shown in Fig. 10 with either diffractive MEMS modulators [72] or analog tilt mirrors [73]. The optical setup is similar to that in Fig. 11 except without circulators. These components typically have $40-80$ channels spaced at 100 or $50 \mathrm{GHz}$ with 6- and 7-dB insertion loss and 20- to 30-dB dynamic range. The most advantageous characteristic of MEMS equalizers is the extremely flat passband transmission profile along with low chromatic dispersion at the edges. This performance was achieved after studying the effects of various mirror geometries [74].

After understanding the effects of mirror profile on dispersion, it became possible to use the same basic component structure as the equalizer to provide channel-by-channel dispersion compensation, although this functionality has yet to be adopted in the deployed network [75].

\section{B. Wavelength Add/Drop Multiplexers}

Wavelength switching allows network operators to use optically transparent components to pass through a network node without detecting and regenerating the data signal, and components that enable this have been the subject of intense research and development. The most basic wavelength switch is the dynamically reconfigurable WADM, which is essentially a $1 \times 2$ or $2 \times 2$ optical switch operating independently on each wavelength channel.

WADM was a natural extension of MEMS equalizers, and the first demonstration of a MEMS add/drop switch based on digital tilt mirrors occurred almost simultaneously with the equalizer [76], [77]. Add/drop requires four ports, twice as many as the equalizer, and so, the basic structure is slightly more complex (Fig. 11). The system is still based on a blazed diffraction grating, which is now illuminated with an upper and lower beam path. The active device is a linear array of 16 digital tilt mirrors fabricated with surface micromachining in the MUMPS process. Each mirror defines a DWDM channel and, in switching, directs the reflected signal back along the input direction or tilted into a new path. Optical circulators on the two I/O fibers separate the forward and reverse propagating signals. The mirrors in this switch tilted by $\pm 5^{\circ}$ under a $20-\mathrm{V}$ signal, switching in $20 \mu \mathrm{s}$. A quarter-wave plate is used to achieve $0.2-\mathrm{dB}$ polarization dependence on a total insertion loss of $7.5 \mathrm{~dB}$.

The DCE is closely related to the WADM, and in fact, it is possible to use high-contrast equalizers as $1 \times 1$ switches in a 


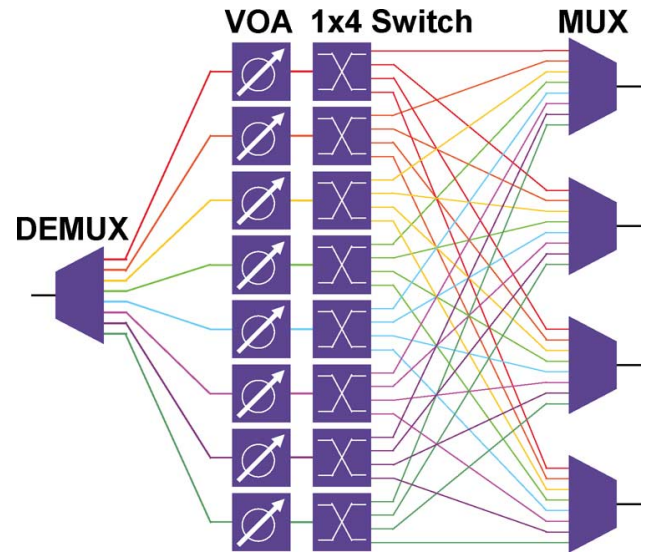

Fig. 12. Equivalent circuit of a $1 \times 4$ WSS. Eight wavelength channels are shown in this example.

"broadcast and select" architecture [78]. The primary disadvantage of this architecture is that it is intrinsically lossy: Signals are power split, and then unwanted signals are blocked before combining into the output fiber. This does allow multicasting, i.e., duplicating signals to multiple output fibers. Broadcast and select was actually the first to be implemented in the network but is generally expected to be phased out in favor of multiport WSSs, which in addition to switching also provide channel equalization [79] with no additional cost or complexity.

\section{Wavelength-Selective Switches (WSSs)}

As optical networks evolve from a simple ring topology with WADM nodes to optical mesh networks, WSSs with more than one output port are needed to link the node to three or four neighboring nodes with each link carrying two-way traffic. The WADM concept can be extended to switches with $N$ output ports, where $N$ is larger than 2 . This is called $1 \times N$ WSS [80]-[82]. Fig. 12 shows the equivalent circuits of a $1 \times 4$ WSS. It consists of a WDM demultiplexer, $N_{\lambda}$ of $1 \times N$ space division switches ( $N_{\lambda}$ is the number of wavelength channels) and $N$ WDM multiplexers. The WSS can be realized by a similar grating spectrometer configuration as the WADM, with the digital micromirrors replace by "analog" ones. A large continuous scan angle is required to direct the output beam to any of the $N$ output fiber collimators. High fill factor is desired to minimize the gaps between wavelength channels. The mirror size is usually several times larger than the focused optical beam to attain a wide and flat passband for minimal signal distortion.

A detailed review paper on WSS was published recently [80]. The optical setup for Lucent's WSS is shown in Fig. 13. The first subassembly maps all fiber I/Os to a common spot (point B), and the second subassembly (resolution lens and grating) separates and focuses the wavelengths onto the micromirror array at the image plane. Tilting of the mirror changes the direction of the reflected beam at point $\mathrm{B}$ and sends the optical beam into a different output fiber. A refined design incorporates anamorphic optics in the input stage to reduce the physical size of the switch while maintaining the same spectral resolution at the expense of longer micromirrors.

Experimentally, $1 \times 4$ WSSs with 128 channels spaced on a $50-\mathrm{GHz}$ grid and with 64 channels spaced on a $100-\mathrm{GHz}$

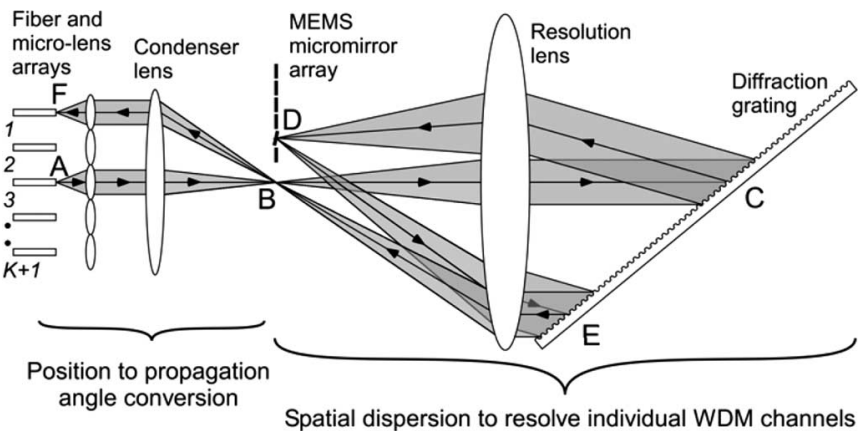

Fig. 13. Schematic optical setup of $1 \times 4$ WSS (reprinted from [80] with permission).

grid have been demonstrated. The typical optical insertion loss ranges from 3 to $5 \mathrm{~dB}$. The channel passband is directly related to the confinement factor, which is defined as the ratio of the mirror size to the Gaussian beam diameter. A confinement factor of $>2.7$ is needed to produce a flattop spectral response with $>74 \%$ passband width measured at $-1 \mathrm{~dB}$ point. JDSU has reported a similar $1 \times 4$ WSS with $3.5-\mathrm{dB}$ insertion loss [81]. UCLA has reported a similar WSS with excellent openloop stability [82].

The analog micromirror array plays a key role in the performance of the WSS. Several types of WSS micromirror arrays have been reported, including electrostatic [83], [84] and electromagnetic [85] actuations. The key parameters are large continuous scan angle and high fill factor, with the mirror size and pitch matching those of the optical system. Lucent employed a fringe-field actuated SOI micromirror array [83] and achieved a mechanical tilt angle of $9.2^{\circ}$ at $175 \mathrm{~V}$. The resonant frequency is $3.8 \mathrm{kHz}$ for $80-\mu \mathrm{m}$-wide mirrors.

More efficient actuation has been obtained using vertical comb drive actuators. Hah et al. reported a low-voltage analog micromirror array for WSS [84]. The schematic and the SEM of the micromirror are shown in Fig. 14. The mechanical structures are completely covered by the mirror; therefore, a high fill factor is achieved along the array direction. The actuation voltage is as low as $6 \mathrm{~V}$ for mechanical tilt angles of $\pm 6^{\circ}$. High resonant frequency $(3.4 \mathrm{kHz})$ and high fill factor $(98 \%)$ are also achieved [86]. The excellent stability of the mirror $\left( \pm 0.00085^{\circ}\right)$ enables open-loop operation of the switch with insertion loss variation of $< \pm 0.0035 \mathrm{~dB}$ over $3.5 \mathrm{~h}$ [82].

Scaling of WSS has been analyzed in [86]. The figure of merit is the ratio of the port count and channel spacing $\left(N / \Delta \lambda_{\mathrm{ch}}\right)$. It is proportional to the product of the effective aperture of the resolution lens and the grating dispersion. Most of the reported WSSs have a port count of four. A larger port count $(N \geq 8)$ is desirable for mesh optical networks, where it is necessary to provide two-way links to three or four adjacent neighboring nodes. The port count can be increased from $N$ to $N^{2}$ by arranging the output collimator in a 2-D array. This is referred to as $1 \times N^{2}$ WSS [86]-[88]. Micromirror arrays providing two-axis beamsteering functions are needed for this architecture. This can be accomplished by using either a linear array of two-axis micromirrors [89], [90] or a pair of oneaxis scanners with orthogonal scanning directions in a $4-f$ configuration [86]. The former is more attractive since the 


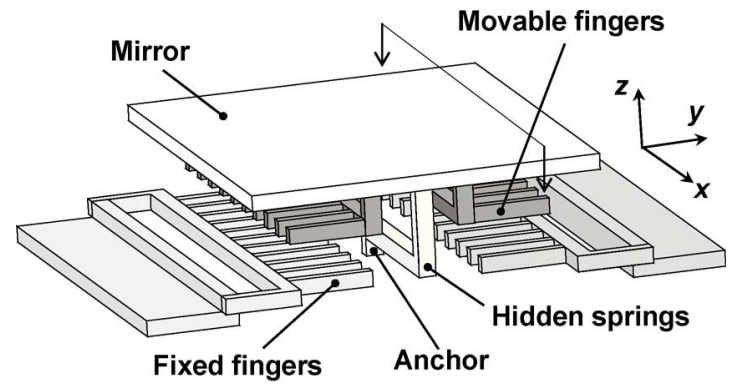

(a)

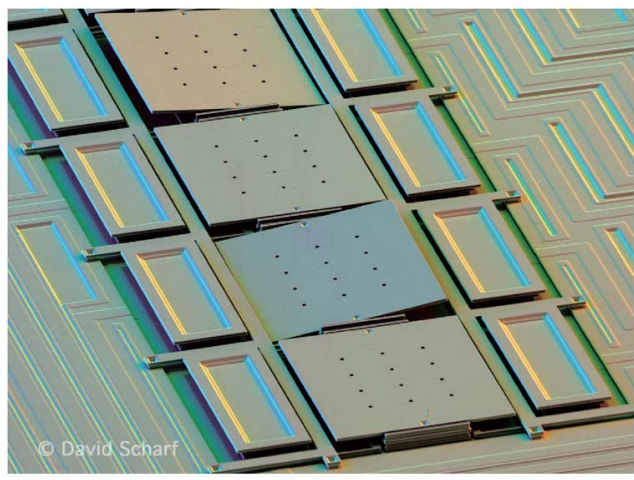

(b)

Fig. 14. (a) Schematic and (b) SEM of the analog micromirror with hidden vertical comb drive actuators (SEM taken by D. Scharf).

optical system is simpler and has twice larger port count. Two types of two-axis analog micromirror arrays have been reported for WSS applications. The first is a parallel-plate-actuated micromirror suspended by cross-coupled torsion springs underneath the mirror [89]. Mechanical scan angles of $\pm 4.4^{\circ}$ and $\pm 3.4^{\circ}$ have been achieved for the two axes at actuation voltages of $\sim 90 \mathrm{~V}$. A $1 \times 14 \mathrm{WSS}(3 \times 5$ collimator array $)$ with $50-\mathrm{GHz}$ channel spacing was been constructed using this mirror array.

Two-axis micromirror arrays with larger scan angles have been demonstrated using vertical motion amplifying levers [90]. The schematic of the mirror is shown in Fig. 15. The mirror is supported by four levers through compliant two-axis torsion hinges. The levers amplified the vertical displacement 3.3 times. Using four vertical comb drive actuators, scan angles of $\pm 6.7^{\circ}$ have been achieved for both axes at $75-\mathrm{V}$ actuation voltages with a fill factor of $98 \%$. The resonant frequency is $5.9 \mathrm{kHz}$. By combining this micromirror array with a densely packed 2-D collimator array, a WSS that is scalable to a port count of $1 \times 32$ (Fig. 16) has been demonstrated [87]. The channel spacing is $100 \mathrm{GHz}$, and the fiber-to-fiber insertion loss is $5.6 \mathrm{~dB}$.

\section{Wavelength-Selective Crossconnects (WSXC)}

WSXCs are desired for mesh-based optical networks. They can reduce the cost of the networks by eliminating the optical-electrical-optical (OEO) conversions. There are several approaches to implement WSXC using MEMS technologies. One approach is to combine separate wavelength demultiplexers such as planar AWG (arrayed waveguide grating) components with wavelength-independent $N \times N$ switches, as shown in Fig. 17. In Fig. 17(a), all the channels can flow through a

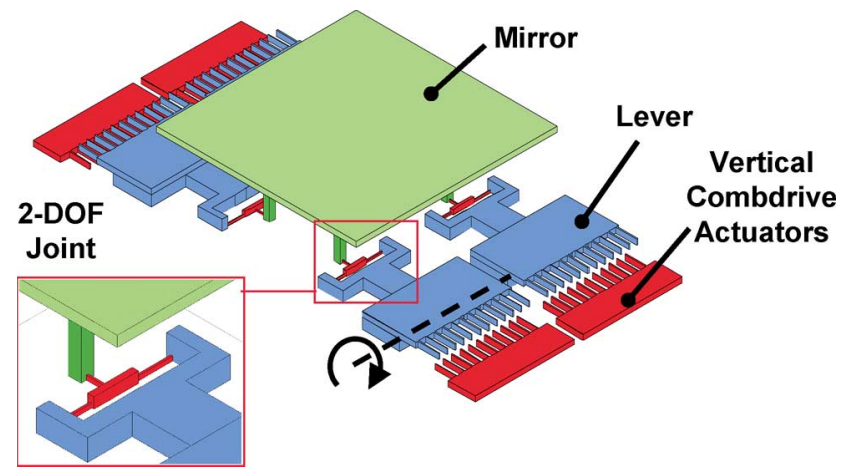

Fig. 15. Schematic of the two-axis analog micromirror array for WSS.

single large switching fabric $(>100 \times 100$ ports, such as the 3-D MEMS crossconnect) [91], [92]. An alternative approach, which is shown in Fig. 17(b), is to use a smaller switch $(8 \times 8$ or $16 \times 16$ ports, such as the MEMS 2-D switch) for each wavelength [29], [34]. In both cases, some of the ports of the transparent switching fabric can be connected to a conventional OEO router to enable higher-level network functionality, such as packet switching, for a limited number of channels.

WSXC can also be constructed by integrating wavelength demultiplexing directly into the free-space optical switch fabric. The simplest of such systems uses passive power splitters to duplicate all DWDM inputs, which are then sent through MEMS wavelength blockers and are essentially high-contrast channelized spectral equalizers, to block unwanted signals from entering passive combiners to the output DWDM fibers [93]. Wavelength blocking WSXCs have large intrinsic splitting and combining loss, which must usually be compensated with an optical amplifier for each fiber port.

The most power-efficient approach to WSXC is to integrate wavelength multiplexing and MEMS multiport switching. This can be done in a single monolithic component [39], [40]. Arguably the most effective approach to WSXC, however, is to use $1 \times N$ WSSs as building blocks [94]. An $N \times N$ WSXC can be realized by interconnecting $N$ modules of $1 \times N$ WSSs and $N$ modules of $N \times 1$ WSSs [Fig. 18(a)]. Alternatively, we can replace the $1 \times N$ WSSs in the first stage with $1 \times N$ passive optical splitters [Fig. 18(b)]. The latter implementation has a fundamental $1 / N$ splitting loss but allows broadcast and multicast functions. This approach was used in the $4 \times 4 \mathrm{WSXC}$ with 64 channels and on a 100-GHz grid [94]. The total insertion loss is $10.5 \mathrm{~dB}$, of which $6.5 \mathrm{~dB}$ comes from the splitter $(0.5 \mathrm{~dB}$ excess loss plus 6-dB splitting loss). In addition to crossconnect, their implementation also provides dynamic spectral equalization and channel blocking capabilities. This approach to WSXC is favored by network operators because it allows flexible provisioning: A fiber node that begins as a simple spectral equalizer can be upgraded to add/drop and then to a full degreefour wavelength crossconnect without interrupting traffic.

\section{E. Spectral Intensity Filters}

Wavelength control is critical to the operation of optical communication systems. WDM fiber optical systems require sources, (de)multiplexers, dispersion compensators, channel 


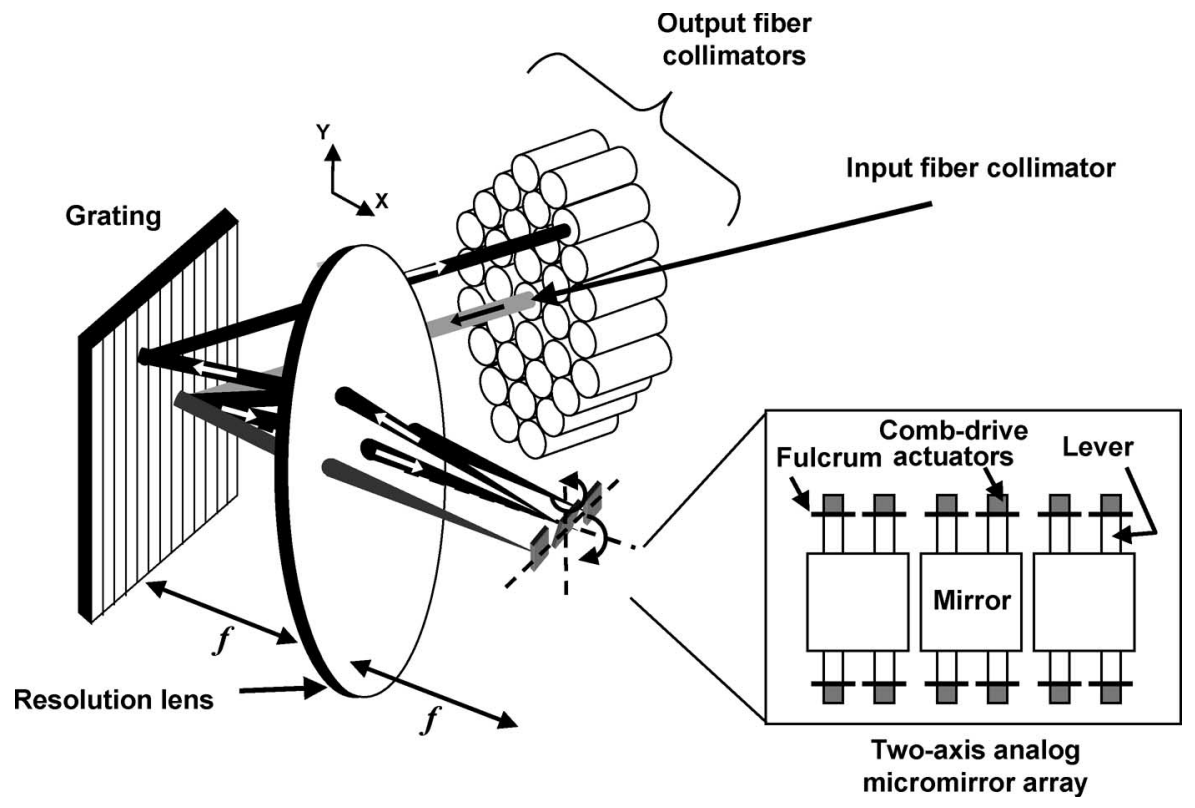

Fig. 16. Schematic setup of the $1 \times 32$ WSS using two-axis micromirror array.

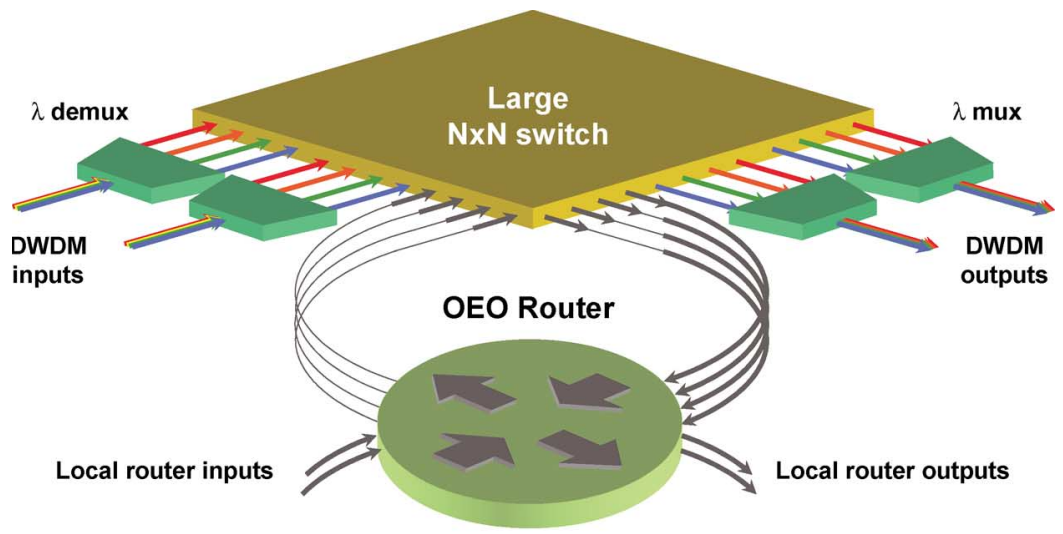

(a)

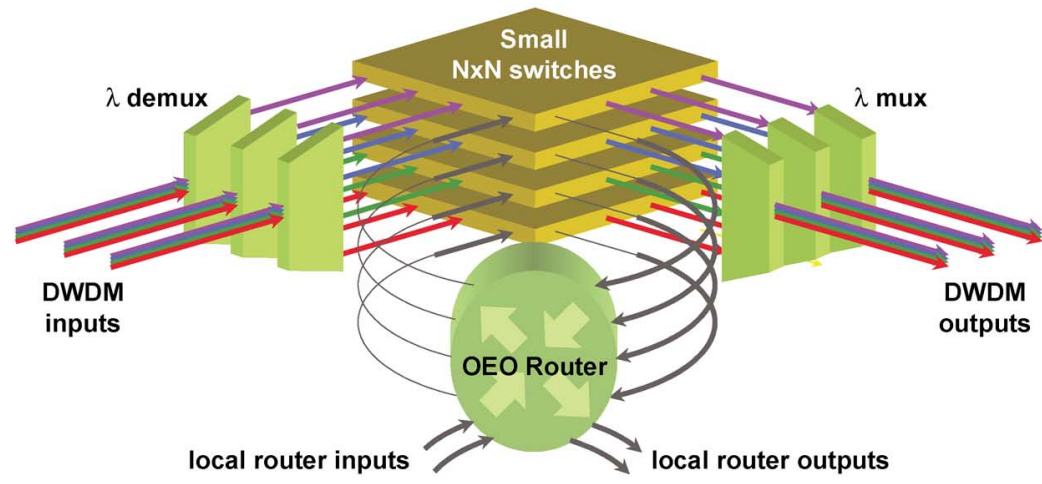

(b)

Fig. 17. WSXC implemented with discrete spectral multiplexers and $N \times N$ switches using (a) one large $N \times N$ switch or (b) multiple small $N \times N$ switches, each dedicated to a single wavelength. The WSS is shown integrated with an OEO router for high-level functionality on a limited subset of channels.

monitors, and receivers with accurate center wavelengths and bandwidths. Optical MEMS adds much needed flexibility to wavelength control by providing tunable optical devices that enable better utilization of the spectrum, reduce the required number of different components to build a system, facilitate communication between different systems, and simplify up- grades. Ultimately, the wavelength agility provided by tunable optical MEMS components and the advantages of miniaturization, integration, and parallel processing lead to communication systems with better performance and lower cost.

Optical MEMS filters and spectrometers come in a large variety of designs. Most traditional optical filters and spectrometers 


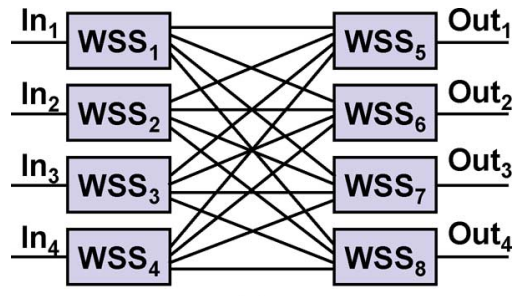

(a)

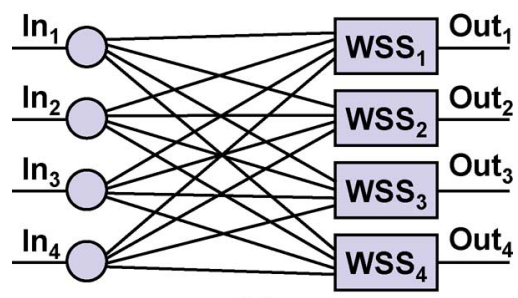

(b)

Fig. 18. A $4 \times 4$ WSXC realized by (a) four $1 \times 4$ WSSs and four $4 \times 1$ WSSs and (b) four $1 \times 4$ passive splitters and four $4 \times 1$ WSSs.

have MEMS counterparts, and in addition, MEMS enables a number of devices that are impractical, if not impossible, to implement in traditional technologies. In this section, we will describe MEMS implementations of traditional filter and spectrometer architectures as well as several designs that rely for their operation on the characteristics of MEMS technology. The objective is not a comprehensive coverage of all optical MEMS filters and spectrometers. Instead, the emphasis is on the advantages and challenges that are unique to the MEMS implementation of device architectures.

A tunable Fabry-Pérot ( $\mathrm{F}-\mathrm{P})$ is relatively simple to implement in MEMS technology. Two single-layer dielectric or semiconductor mirrors, or a movable single-layer mirror and a stationary highly reflective multilayer mirror, are sufficient to create a low-finesse F-P that can be tuned by moving the mirrors relative to each other by electrostatic or other types of MEMS actuators. This type of F-P is of limited use due to the broad reflection and transmission bands resulting from the low reflectivity of single-layer dielectric and semiconductor mirrors. In principle, we can reduce the filter bandwidth by making the cavity longer, but that is counter productive since miniaturization is one of the major motivations for using MEMS technology. In addition, there are many applications, e.g., channel filters in WDM fiber optic communication systems, where the important figure of merit is the finesse or the ratio of the free spectral range (FSR) to the transmittance bandwidth rather than the transmission bandwidth. Finesse is determined solely by the mirror, as can be seen from the standard formula for lossless F-Ps

$$
\text { Finesse } \equiv \frac{\Delta f_{\mathrm{FSR}}}{\Delta f_{\mathrm{FWHM}}}=\frac{\pi \sqrt{\sqrt{R_{1}} \sqrt{R_{2}}}}{1-\sqrt{R_{1}} \sqrt{R_{2}}} \approx \frac{\pi}{1-R}
$$

where $R_{1,2}$ are the reflectivities of the two mirrors. Most applications require higher finesse than can be obtained with single-layer dielectric mirrors to achieve acceptable specifications. Until the arrival of photonic crystals, which will be discussed in a later section of this paper, high-finesse F-Ps could only be fabricated using multilayer dielectric mirrors.
To be movable by MEMS actuators, these multilayer dielectric mirrors have to be free standing and are therefore not supported by the rigid substrates that are traditionally used. This presents challenges in MEMS fabrication due to the thermal stresses that build up in the mirrors stacks, leading to the temperaturedependent mirror curvature that is unacceptable for high-finesse applications. This fabrication challenge has been met through a variety of approaches. Early work [95] used the full thickness of silicon wafer to provide a solid substrate. These devices were fabricated by wafer bonding and were relatively bulky. Smaller devices have been created by using free-standing $\mathrm{Si}-\mathrm{SiO}_{2}$ mirror stacks, but these mirrors have some problems with curvature [96]. By careful compensation of the material stress in the dielectric stack, silicon-compatible free-standing dielectric mirrors with better than $99 \%$ reflectivity have been demonstrated [97].

A very elegant and powerful approach is to grow latticematched semiconductor mirrors, most typically using molecular beam epitaxy. Early work in AlGaAs [98], [99] has led to the rapid development of this field with several important contributors [100]-[102], and it has also led to the creation of MEMS tunable vertical cavity surface emitting semiconductor lasers (VCSELs) (for an in-depth description of MEMS tunable VCSELs, see [103]). This type of fabrication process results in excellent mirrors, but the process is not compatible with silicon technology.

An approach that avoids the complications of bending due to thermal stress in free-standing dielectric stacks is to tune the filters thermally rather than by mechanical motion. In such thermally tuned devices, the dielectric mirrors are deposited directly on a silicon substrate with an intermediate film of thermooptical material. The temperature and therefore the effective optical thickness of the material between the dielectric mirrors are controlled by thermal dissipation in integrated resistors. This approach has been used to create tunable channel-dropping WDM filters with narrow transition bands [104].

\section{F. Dispersion Compensators}

In contrast to channel selection, dispersion compensation in WDM systems does not require high out-of-band suppression, so low-finesse F-P provides sufficient dispersion for most fiber communication systems. To avoid unwanted amplitude variations, dispersion compensation is typically carried out with Gires-Tournois (G-T) interferometers [105]. The G-T interferometer is an F-P with a highly reflective back mirror. In the ideal case of plane wave incidence and a $100 \%$ reflective back mirror $\left(r_{2}\right)$, the reflectance is always unity, so the ideal $\mathrm{G}-\mathrm{T}$ is an allpass filter with a strong phase variation around resonance.

Fig. 19 shows a G-T based on the MARS device discussed in Section II-A [5]. The MARS device is a low-finesse G-T with a highly reflective dielectric stack as the back mirror and a single free-standing $\lambda / 4$ silicon-nitride film as the front mirror. This device performs very well as a dispersion slope compensator in spite of the relatively low finesse. A linear dispersion tunable from -100 to $100 \mathrm{ps} / \mathrm{nm}$ over $50 \mathrm{GHz}$ in C-band has been experimentally demonstrated [106]. 

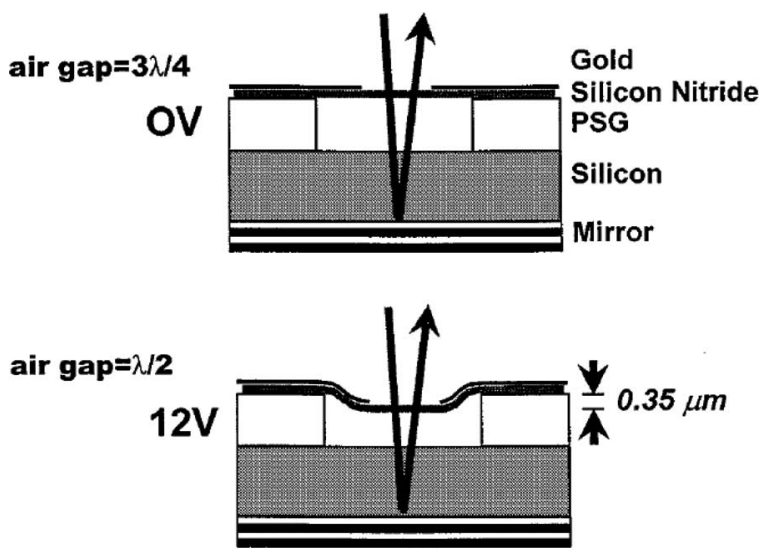

Fig. 19. MEMS allpass filter schematic showing the change in air gap with applied voltage (reprinted from [5] with permission).

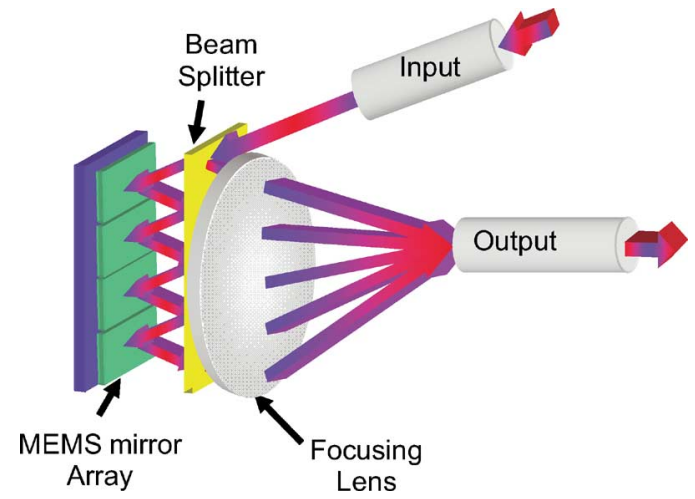

Fig. 20. Schematic diagram of the MEMS G-T interferometer.

A variation of the $\mathrm{G}-\mathrm{T}$ interferometer operates on oblique incidence so that the optical beam follows a zigzag pattern and the reflections from the back mirror are spatially separated, as shown in Fig. 20. The output from this device is the interference pattern of the first reflected beam and the partially transmitted beams from the front mirror. This geometry allows the phase of the reflections to be individually modulated and enables tuning of a variety of filter characteristics. Tunable (de)interleavers [107], amplitude filters [108], and dispersion compensators with linear dispersion tunable from -130 to $150 \mathrm{ps} / \mathrm{nm}$ over $40 \mathrm{GHz}$ in C-band [109] have been demonstrated. This variation of the G-T interferometer is not an allpass filter, even in the idealized case, so careful attention has to be paid to avoid parasitic amplitude modulation when the phase is tuned.

\section{G. Transform Spectrometers}

Transform spectrometers also lend themselves to MEMS implementations, and several different architectures have been demonstrated. Fig. 21 illustrates a design that uses a traditional Michelson interferometer, in which the movable mirror is actuated by an electrostatic comb drive [110]. The light from the source is split into two parts by a beam splitter, and the two parts are reflected from two different mirrors, one of which is movable to create a variable path length for the tow part of the incident light. After reflection, the two parts of the incident light recombine and interfere on the beam splitter. Each wavelength

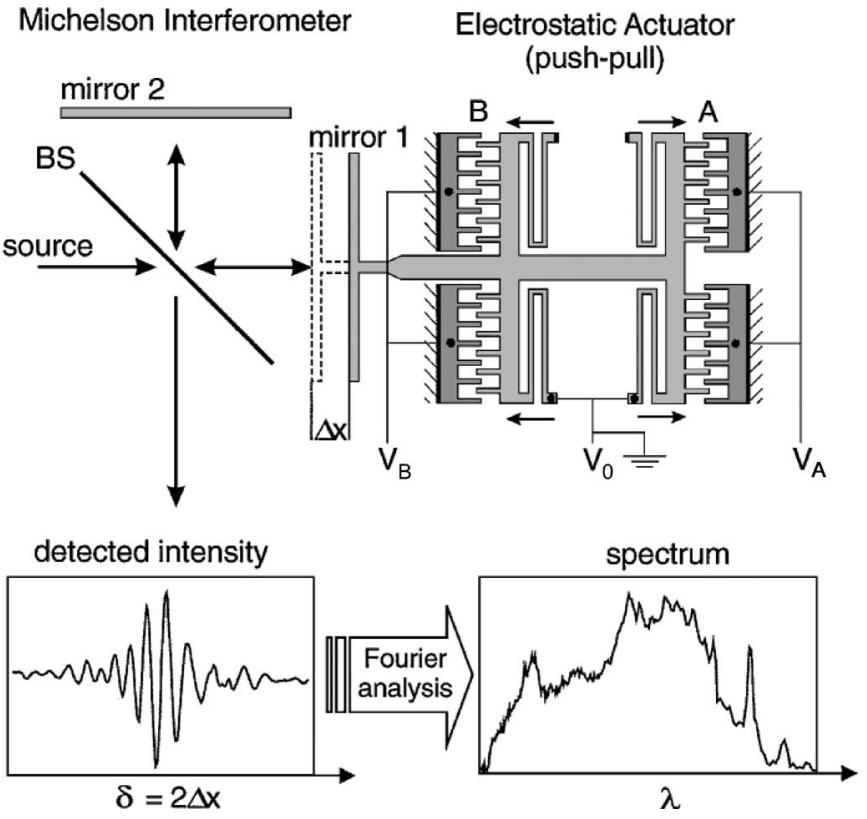

Fig. 21. Schematic of a Fourier transform spectrometer based on a traditional Michelson interferometer with a MEMS electrostatic actuator (reprinted from [110] with permission).

of the detected optical power or intensity $P_{\text {detected }}$ shows a harmonic dependence on the path-length difference $\Delta x$, i.e.,

$$
P_{\text {detected }}=P_{\text {incident }} \cdot \cos \left[\frac{4 \pi \cdot \Delta x}{\lambda}\right]
$$

where $P_{\text {incident }}$ is the incident optical power, and $\lambda$ is the wavelength. The Fourier transform of the optical spectrum is obtained by varying the path-length difference $\Delta x$, and the spectrum is found through an inverse Fourier transform.

Common to all transform spectrometers, the spectral resolution $\Delta \lambda_{\mathrm{FWHM}}$ is determined by the total range of motion $\Delta x_{\max }$ of the moving mirror [111]

$$
\frac{\Delta \lambda_{\mathrm{FWHM}}}{\lambda}=0.5 \cdot \frac{\lambda}{\Delta x_{\max }} .
$$

This simple equation highlights the main challenge in the MEMS implementations of transform spectrometers. Because the spectral resolution is inversely proportional to the maximum actuation distance that can be achieved, long-travel actuators are required. The micrometer-scale displacements that are sufficient for many MEMS applications are not useful here, and even long-range MEMS actuators, e.g., electrostatic comb drives with several tens of micrometers of motion, achieve only modest resolutions. The challenge in implementing MEMS transform spectrometers with good resolution therefore boils down to the creation of fast, accurate, and reliable long-range actuators. Transform spectrometers are also relatively complex systems with several optical components that must be well aligned. This represents both a challenge and an opportunity for MEMS. It is difficult to fabricate several very different optical devices in the same MEMS process, but if it can be done, the accuracy of MEMS technology simplifies alignment and packaging. 


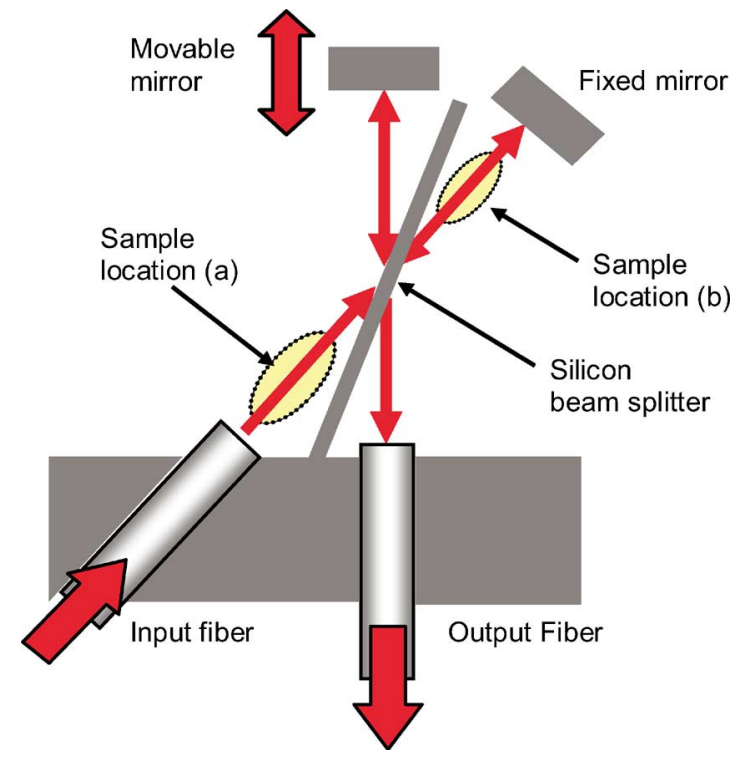

Fig. 22. Schematic of single-chip integrated transform spectrometer based on vertical micromirrors with integrated MEMS actuators. The nonnormal incidence on the beam splitter is due to the restrictions of the surfaces that can be defined by anisotropic etching of $\mathrm{Si}$.

One approach to high-resolution transform spectroscopy with MEMS is based on "microjoinery" [112]. The microjoinery spectrometer utilizes the precision of bulk micromachining to establish a very accurate and long range path for a slider that carries the moving reference mirror of the interferometer. The strength of this solution is that the reference mirror can be moved over long distances to create a spectrometer with very good spectral resolution. The challenge is to integrate a suitable actuator that provides the motion over the full range of the track established by the microjoined slider. Using magnetic actuation with external magnetic fields, motion of several centimeter has been demonstrated, resulting in fractional resolution on the order of $10^{-5}$ in the visible wavelength range.

Transform spectrometers with modest resolution can be integrated on a single chip by using vertical mirrors with integrated actuators [113]. The single-chip integrated transform spectrometer shown in Fig. 22 is implemented through a combination of anisotropic etching and deep reactive ion etching (DRIE). Anisotropic etching is, as the name implies, dependent on crystalline orientation, i.e., it etches different crystalline planes at different etch rates, resulting in very smooth surfaces that can be used as optical interfaces and mirrors. DRIE is used to create electrostatic actuators and fiber grooves that should not be restricted by the crystalline orientation of the silicon. In the implementation of the architecture shown in Fig. 22, the beam splitter and the movable mirror are both defined using anisotropic etching, while the fixed mirror is defined by DRIE. It is also possible to use a combination of two anisotropically etched mirrors instead of the DRIE-defined fixed mirror.

The transform spectrometers described so far are of the traditional Michelson interferometer design. The characteristic advantages and challenges of MEMS technology have inspired nontraditional solutions of different kinds. One such MEMS architecture is shown in Fig. 23, which depicts a reflection phase grating with a variable grating amplitude [114]. The

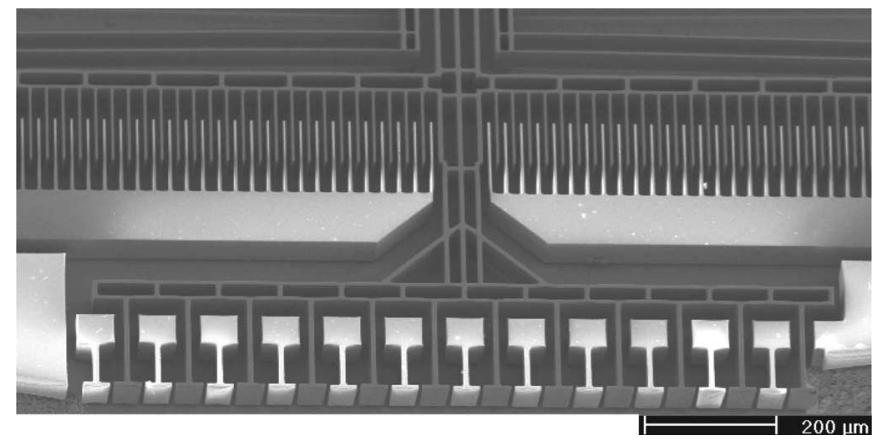

Fig. 23. Transform spectrometer based on a diffraction phase grating with a tunable grating amplitude. The grating consists of alternating fixed (light) and movable (dark) mirror elements. The movable mirrors are displaced by an electrostatic actuator to create a variable path-length difference (reprinted from [114] with permission).

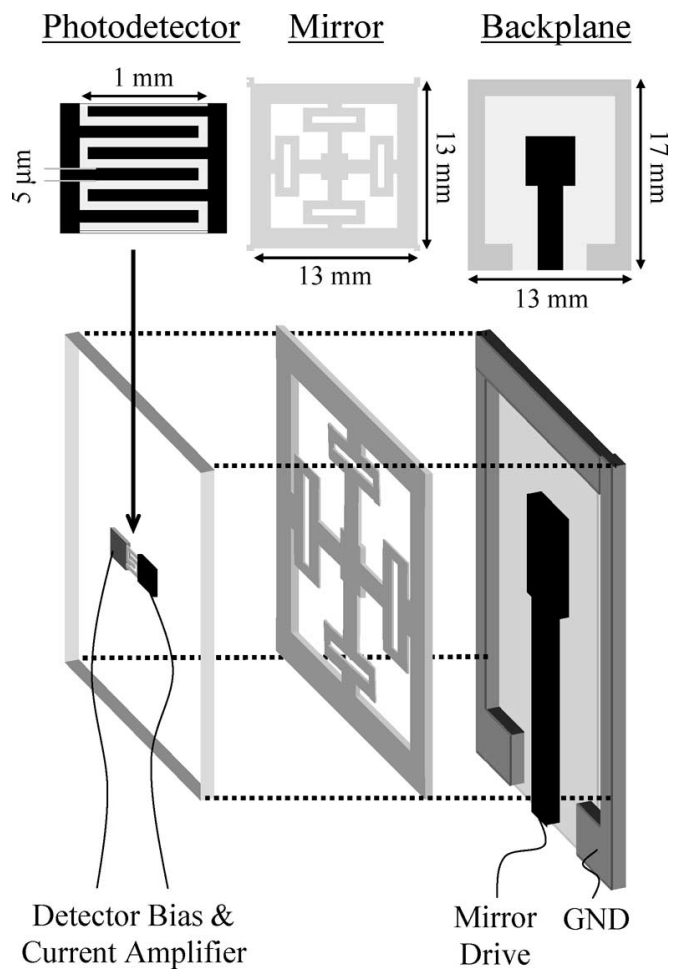

Fig. 24. Transform spectrometer using a semitransparent detector in a standing wave cavity (reprinted from [115] with permission).

reflected optical power from the grating has a harmonic dependence on the grating amplitude, just like the dependence of traditional Fourier transform spectrometers on the optical pathlength difference. The grating transform spectrometer maps readily onto the more traditional Michelson structure. The main conceptual difference is that the grating acts both as a beam splitter and as a two-beam interferometer with a variable pathlength difference.

In the spectrometer shown in Fig. 24, a standing wave is being sampled at one spatial location by a semi-transparent detector [115]. The position of the standing wave pattern is varied by moving the rear mirrors of the standing-wave cavity. The response of this spectrometer is again a harmonic function of the mirror displacement, leading to the same dependence 


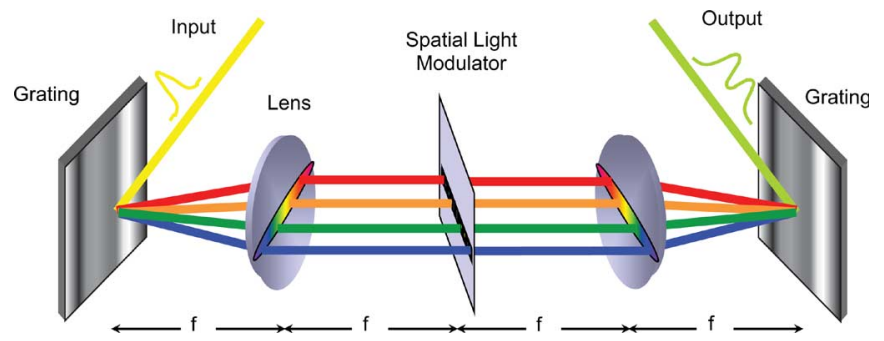

Fig. 25. Basic optical system for spectral synthesis and measurements. The incident light is collimated onto a diffraction grating and dispersed on SLM. The modulated spectral components from the SLM are recombined on the grating and focused on the output, which can be an optical fiber, a detector, or a detector array. The SLM is shown here as a transmission device, but it is more common to use a reflective SLM in MEMS applications.

of resolution on mirror displacement as in other transform spectrometers.

The two implementations in Figs. 23 and 24 show how the flexibility of MEMS technology enables nontraditional solutions. Both of these implementations are very compact, thereby facilitating miniaturization, integration, and packaging. Neither of the two achieves better than modest resolution due to the limited maximum displacement of their actuators.

\section{H. Diffractive Spectrometers and Spectral Synthesis}

The nontraditional implementations of transform spectrometers described above illustrate one of the major strengths of optical MEMS. The flexibility, complexity, and accuracy afforded by lithography enables architectures that cannot practically be created by traditional manufacturing technologies. Another advantage of optical MEMS is the ability to create spatial light modulators (SLMs) and other devices that require large numbers of identical components. This attribute has been exploited to expand the functionality of grating spectrometers. A traditional grating spectrometer measures spectral amplitude by dispersing the wavelengths of the incoming light over a range of angles. The spectral amplitude can be measured by using an array of detectors or by rotating the grating and using a single detector. A variation of this traditional concept is to place an SLM in the back focal plane of the lens that captures the dispersed light from the grating, as shown in Fig. 25.

In this device, the spectral components of the incident are dispersed by the grating and modulated by the SLM. The SLM may modulate the amplitude or phase, or both, of the dispersed light. This very versatile configuration can therefore be used for Hadamard spectroscopy [116], optical pulse shaping [117], [118], spectral phase measurements [117], adjustable time delays [119]-[121], wavelength-selective optical WDM switches [40], WDM add/drop filters [77], and a wide variety of other applications. The SLMs used in the architecture must be tailored to the specific applications. The flexibility in size, form, and function of optical MEMS has made it the technology of choice for a large number of these applications.

A variation of the grating spectrometer that uses optical MEMS not as an SLM to modulate dispersed light but as the dispersing element is shown in Fig. 26. The idea here is to deform the SLM, which here acts as a grating or dispersive element, to dynamically change the characteristics of the filter

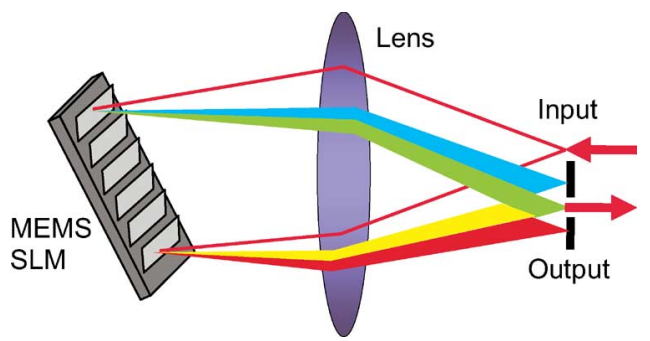

Fig. 26. Optical MEMS SLM as a diffractive element for synthesis of spectral amplitude and phase. The SLM is deformed to create a surface that diffracts the desired spectral components of the incident light into a specific output.

or spectrometer. This architecture is neither as powerful in terms of spectral manipulation nor as efficient in terms of optical throughput as the one shown in Fig. 25. It does, however, require fewer components and is more compact, which makes it preferable for many practical systems, including displays [122]-[124], WDM variable attenuators [20], interferometric displacement sensors for a variety of applications [125]-[129], spectral synthesis [130], and compact optical filters [131] and pulse shapers [132].

The diffractive MEMS device shown in Fig. 26 is conceptually similar to an adaptive optics (AO) mirror [133]. In AO, a deformable surface is employed to compensate for aberrations imposed on an optical wavefront by inhomogeneities in the transport medium between the source and the detector. Most, if not all, filter applications require much more wavelength dispersion than can be provided by $\mathrm{AO}$ mirrors that are designed for wavefront corrections. This can be understood by considering the impulse response of the filter; the output is an impulse train corresponding to the height distribution of the individual reflectors of the diffractive surface. Neglecting weak wavelength dependencies in diffraction efficiency and output coupling, the transmission of the filter is given by the Fourier transform of the impulse response, which in turn is determined by the height distribution of the diffractive surface [134].

This simple conceptual picture of diffractive filter operation leads to three insights that are of importance to MEMS implementations. First, the filter transfer function is the Fourier transform of a nonnegative sequence, which means that in principle any transfer function can be synthesized to within a constant (see [135] for details on the restrictions on synthesized transfer functions).

Another observation we can make from Fig. 26 is that the total length of the impulse response is given by the maximum difference of positions of the reflectors of the diffractive MEMS along the optical axis. The spectral resolution of the filter is therefore inversely proportional to the height difference of the MEMS SLM along the optical axis. For most applications, the resolution specifications require the height to be much larger than the height of practical MEMS structures by themselves; therefore, grazing incidence and large diffraction angles are necessary. Early MEMS diffractive filters that were designed for normal incidence [136] are therefore useful only for lowresolution applications. Better resolution can be obtained by adding another diffractive element [137] or by creating a diffractive structure with high diffraction angle [138]. 
The third characteristic of diffractive filters that is illustrated in Fig. 26 is that the loss of the filter is proportional to its complexity. The incident light is split into $N$ spatially separate channels and then recombined into a single output channel, leading to a $1 / N$ loss. This is true for any optical system that separate the input into $N$ equal channels that are incoherently recombined to create a single output. This means that only wavelengths that are reflected in phase from all $N$ grating elements are completely transmitted by the filter. In other words, to have high optical throughput, the filter must essentially act as a grating with all grating elements acting in phase in the optical passband. If good spectral resolution is also required, the diffractive element must have a high diffraction angle as discussed before. To achieve both high throughput and good resolution, the diffractive element should behave much like a blazed grating and operated such that all the reflectors of the MEMS device are in-phase in the optical passband. Such diffractive MEMS has been demonstrated as amplitude filters [139] and tunable WDM interleavers [140].

\section{Tunable Lasers}

In the filter implementations described in this section, MEMS provides a means to fabricate optical components as well as a substrate for integration and packaging. It is clear from these filter implementations that one of the main advantages of optical MEMS is the opportunity for system-level integration. One of the successful systems applications that utilize optical filters is tunable lasers. VCSELs with tunable cavity length were mentioned above. Here, we will describe MEMS implementations of traditional external cavity semiconductor diode lasers (ECSDLs).

A typical ECSDL has a semiconductor gain medium with a single-mode waveguide. The front facet of the gain medium is anti-reflection (AR) coated, and the output of the singlemode waveguide is collimated onto a diffraction grating. The incident optical mode on the grating is retroreflected back into the waveguide from an external cavity. This setup is the traditional Littrow configuration, as shown in Fig. 27(a). An alternative design, known as the Littman configuration, is shown in Fig. 27(b). Here, the incident light on the rating is diffracted onto a mirror that retroreflects the light via the grating back to the waveguide to create the optical cavity. The advantage of the Littman configuration is that the light is diffracted from the grating twice per round trip of the cavity, leading to better outof-band suppression in the grating filter.

The laser systems in Fig. 27 create two interacting filters, namely 1) the cavity itself with an FSR that is determined by the cavity length and 2) the grating that only reflects one wavelength in the correct direction to establish retroreflection. To obtain lasing without an excessively high pumping threshold, these two filters must be aligned in wavelength, which means that the cavity length has to be controlled with subwavelength accuracy. Accurate alignment and cavity length control are therefore necessary and motivate the use of MEMS technology.

To tune the laser wavelength, the grating is rotated so that the center wavelength of the grating filter is changed. To achieve continuous mode-hop-free wavelength tuning, the grating must

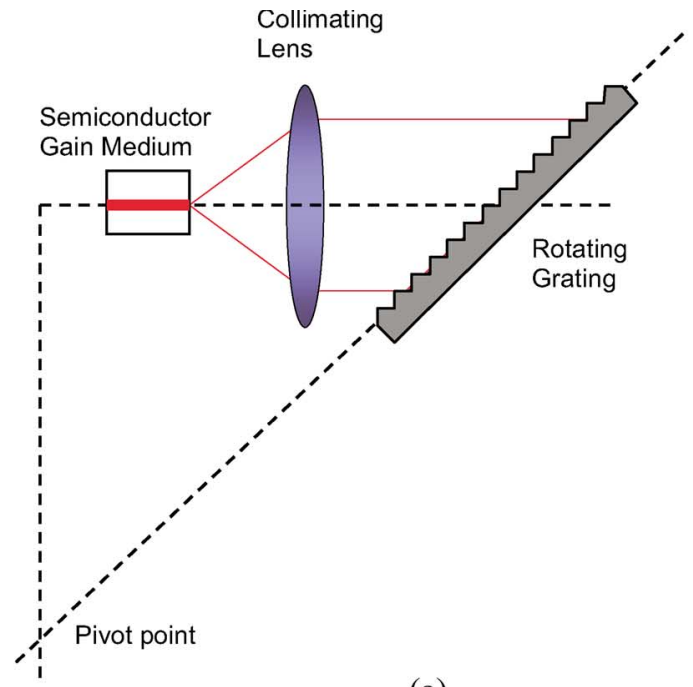

(a)

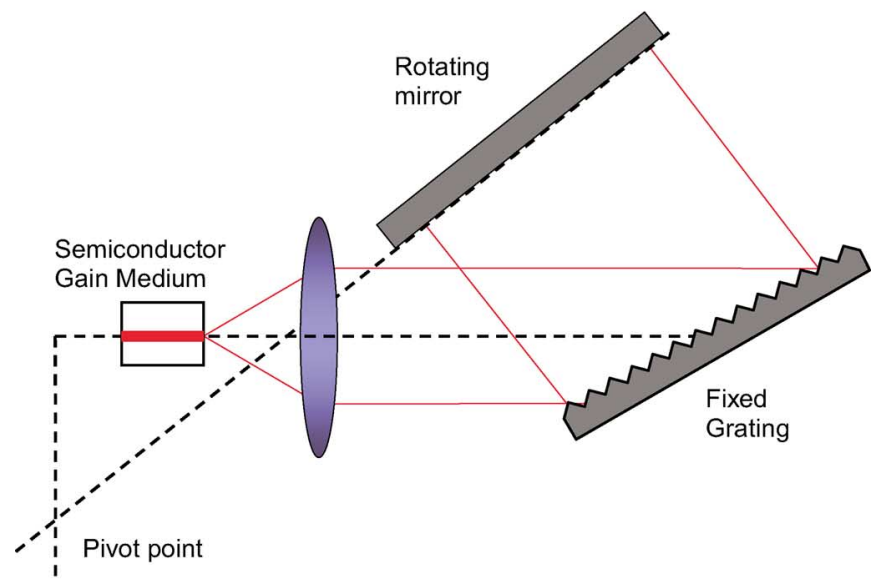

(b)

Fig. 27. Schematic diagrams of the traditional (a) Littrow and (b) Littman configurations of tunable external cavity lasers.

be rotated and translated so that the cavity mode stays aligned with the grating filter. It is well known that if the grating (or mirror in the case of the Littman configuration) is rotated around a pivot point located at the intersection of the line through the rotating surface, and the normal to the optical axis at a point that is a distance $n \cdot \lambda_{v a c}$ from the rotating element along the optical axis, where $n$ is the number of wavelengths in the cavity, and $\lambda_{v a c}$ is the vacuum wavelength, then the cavity mode and the grating filter stay aligned during rotation [141], [142].

In principle, an ECSDL can therefore be controlled by an actuator with one degree of freedom of motion. In practice, at least one extra degree of freedom is required to initially align the cavity mode and grating filter and to compensate for dispersion in the optical components of the cavity. Academic research on MEMS implementations of Littrow [143], [144] and Littman [145] ECSDLs has focused on the development of accurate one-degree-of-freedom actuators that can provide stable mode-hop-free tuning after initial alignment. An interesting alternative is to use a diffractive element with separate phase and amplitude control to avoid macroscopic motion in the external cavity [146]. 


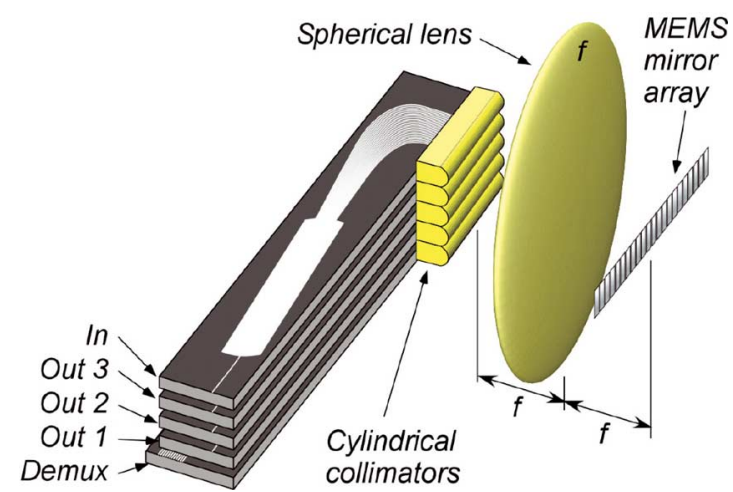

Fig. 28. Schematic of a $1 \times 3$ WSS with hybrid integration of PLC and MEMS (reprinted from [153] with permission).

In contrast to academic research, commercial developments, which have mostly adopted the Littman configuration to achieve better side-mode suppression, have incorporated two or more degrees of freedom in the actuator design so that both initial alignment and compensation for cavity dispersion can be controlled by the MEMS structure [147], [148]. These types of lasers have excellent stability and optical characteristics. The complexity of the optical hardware and the control circuitry lead to costs that are significantly higher than for fixedwavelength semiconductor lasers, however, so systems solutions that use single fixed-wavelength lasers are still preferred even in applications that would benefit from wavelength tuning. One solution with intermediate complexity and proven market potential is to use a set of fixed-wavelength semiconductor lasers and select the output of the one with the most appropriate wavelength using a MEMS mirror for the selection [149].

\section{INTEGRATION OF PLCS AND MEMS}

\section{A. Hybrid Integrated Systems}

The discussion so far has focused on free-space optical systems. Planar lightwave circuits (PLC), on the other hand, allow many WDM functions to be monolithically integrated on a chip. For example, $2 \times 2$ WSXC with 16 wavelength channels [150] and $1 \times 9$ WSS with eight channels and 200-GHz spacing [151] have been reported using thermal optic switches. The main drawback of thermal optic switch is high power consumption and slower switching time. These are the areas where MEMS offers significant advantages. Therefore, hybrid integration of PLC and MEMS could lead to more compact higher functional systems with low-power consumption and fast switching time.

Marom et al. reported a hybrid WSS by combining the silica PLC and the MEMS tilting mirror array [152], [153]. Fig. 28 shows the schematic of a $1 \times 3$ hybrid WSS at $100-\mathrm{GHz}$ spacing [153]. The system consists of five silica PLCs arranged in a vertical stack, each containing an AWG with one star coupler terminated at the PLC edge. The bottom one is used as a demultiplexer for the detection of locally dropped channels. An external spherical lens focuses the dispersed light to the micromirror array. The mirrors tilt in the vertical plane for switching the signals between PLCs. An insertion loss ranging from 5 to $6.8 \mathrm{~dB}$ was measured using a bulk mirror. The hybrid WSS reported by Ducellier et al. employs a two-axis micromir- ror array to steer optical beams both within a PLC (horizontally) and across vertically stacked PLCs (vertically) [154]. Using two PLCs with five AWGs each, a $1 \times 9$ WSS has been realized. The WSS has an insertion loss of 2.8-4.3 dB for the best port and 5.6-7.8 dB for the worst port. The polarization-dependent loss (PDL) of the device is typically $0.3 \mathrm{~dB}$, and the isolation is typically greater than $35 \mathrm{~dB}$ over $\pm 12.5 \mathrm{GHz}$.

Using a different optical system, a $2 \times 2$ WSXC with 36 wavelength channels was realized by butt coupling four stacked PLC chips with a $36 \times 36$ array of two-axis micromirrors [92]. The MEMS array would allow $18 \times 18$ ports with 36 wavelengths. However, the optical loss is high $(20 \mathrm{~dB})$ because the optical axis of the steered beam is not aligned with the receiving PLC waveguide. Another drawback is the required upfront investment of a large switching fabric. Yet another WSXC was reported by using a single arrayed waveguide lens with three diffraction order outputs $(-1,0,+1)$ in conjunction with an array of MEMS piston mirrors [155]. A $2 \times 2$ WSXC with 16 channels on a $100-\mathrm{GHz}$ grid was achieved using circulators for both I/O waveguides. The insertion loss is $10.6 \mathrm{~dB}$.

Other hybrid integrated PLC-MEMS includes a tunable dispersion compensator with $\pm 500 \mathrm{ps} / \mathrm{nm}$ tuning range and 100-GHz FSR using a PLC and a deformable membrane [156]. Wavelength-independent $1 \times N$ optical switches with external [157] and monolithically integrated cylindrical lens [158] have also been demonstrated using a one-axis tilting mirror.

\section{B. Monolithic WSS and WSXC}

Hybrid integrated systems still require bulk lenses between the PLC and the MEMS micromirrors for collimation and focusing. Free-space propagation (length $\sim$ focal length of the bulk lens) is often needed to perform Fourier transformation of the optical beams [152]-[154]. Optical alignment is still necessary. A more compact system can be achieved by monolithically integrating the PLC and the MEMS micromirrors on the same substrate.

Chi et al. have reported a fully integrated $1 \times 4$ MEMS WSS for coarse wavelength-division-multiplexing (CWDM) networks with 20-nm channel spacing [159]. The schematic of the WSS is shown in Fig. 29(a). Like its free-space counterpart discussed earlier, light from waveguide is first collimated by a parabolic mirror, dispersed by a transmission micrograting, and then focused onto the vertical MEMS micromirrors. The only difference is that light is confined vertically in the silicon slab. The lenses in free-space systems are replaced by TIR mirrors. The etched sidewalls form the surfaces of the MEMS micromirrors. All the optical and MEMS components are monolithically integrated on an SOI substrate with a 5- $\mu \mathrm{m}$-thick device layer. The SOI platform is attractive because they are compatible with Si PLC [160] as well as SOI-MEMS [24] technologies. All optical paths are defined by photolithography, and no optical alignment is necessary. Theoretical calculation shows that a 4.1-dB insertion loss is achievable. The $1 \times 4 \mathrm{CWDM}$ WSS chip with eight channels has an area of $1.4 \times 2 \mathrm{~cm}^{2}$. A switching time of less than $1 \mathrm{~ms}$ has been achieved.

The entire WSXC can also be monolithically integrated on a chip using the SOI PLC-MEMS technology [161]. The 


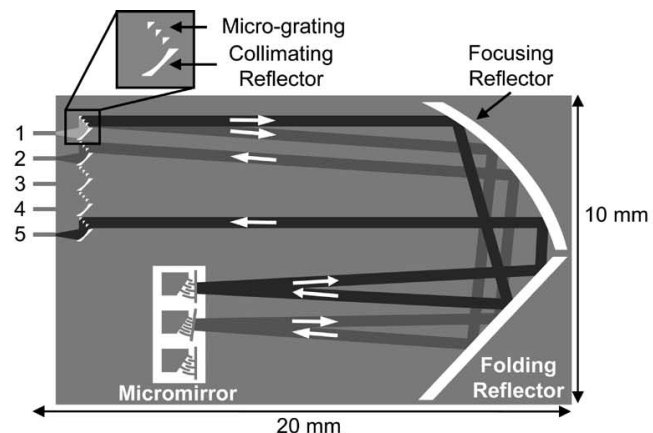

(a)

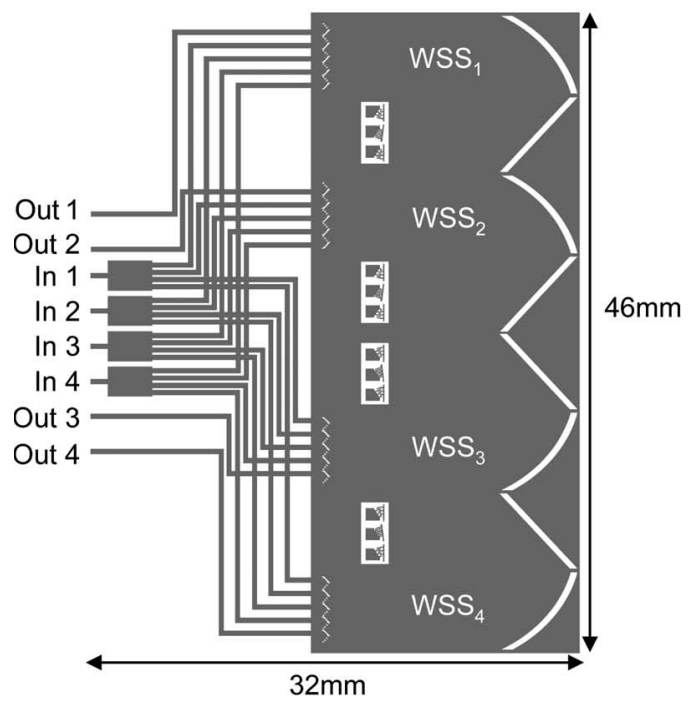

(b)

Fig. 29. Schematic of (a) monolithic $1 \times 4$ WSS and (b) monolithic $4 \times 4$ WSXC realized in SOI MEMS PLC platform.

$4 \times 4 \mathrm{WSXC}$ is realized by interconnecting four $1 \times 4$ splitters and four $4 \times 1$ WSSs with an integrated waveguide shuffle network [Fig. 29(b)]. The $1 \times 4$ multimode interference splitter is $890 \mu \mathrm{m}$ long and $40 \mu \mathrm{m}$ wide. The shuffle network employs $90^{\circ}$ waveguide bend and crossing to minimize loss and crosstalk. The $4 \times 4 \mathrm{WSXC}$ with CWDM grid has an area of $3.2 \times 4.6 \mathrm{~cm}^{2}$. The fiber-to-fiber insertion loss was measured to be $24 \mathrm{~dB}$, which includes the 6-dB splitting loss. The excess loss can be reduced to below $3 \mathrm{~dB}$ by improving the fabrication process.

\section{EMERGing MEMS TECHNOLOGIES AND APPLICATIONS}

\section{A. MEMS Tunable Microdisk/Microring Resonators}

Microdisk or microring resonators offer another order of magnitude size reduction for a wide range of WDM functions, such as add/drop multiplexers [162], dispersion compensators [163], modulators [164], and WDM lasers [165]. Semiconductor microresonators with high index contrast can further reduce the resonator dimensions, producing wide FSRs and small footprints [166]. Integrating MEMS with microresonators will enable a host of tunable WDM functions [167]-[169]. Compared with other tuning mechanisms (thermal tuning [170], [171], electrical carrier injection [172], electroabsorption [173], or

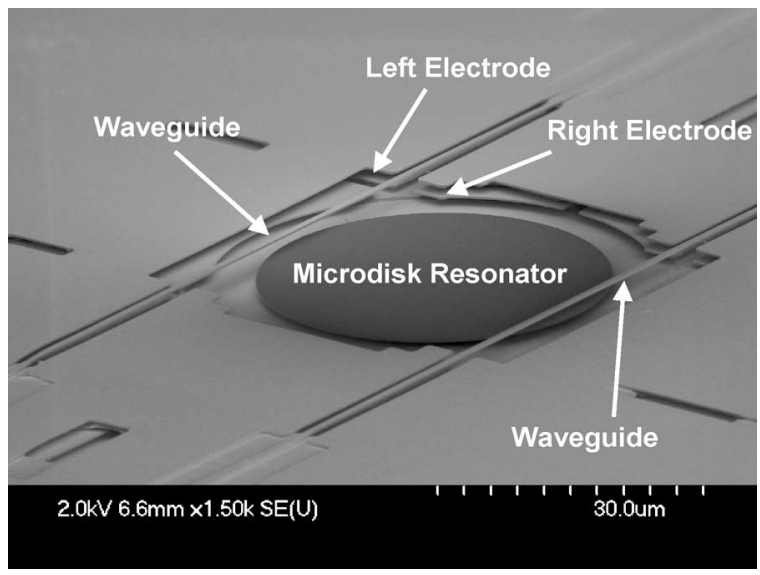

Fig. 30. SEM of MEMS microdisk resonator with variable optical couplers (reprinted from [168] with permission). The suspended waveguides can be deformed by electrostatic actuation, which change the gap spacing between the waveguide and the microdisk.

gain trimming [174] in III-V semiconductors), MEMS tuning is more efficient and consumes much less power. The ability to physically change the spacing between the waveguide and the microresonator enables us to control the coupling coefficient, which is an important tuning parameter for most signal processing functions but difficult to achieve by conventional means.

Lee and $\mathrm{Wu}$ reported a silicon tunable microdisk resonator with tunable optical coupling using MEMS actuators [168]. The SEM of the device is shown in Fig. 30. This is a vertically coupled microdisk resonator with suspended waveguides around the microdisk. The optical coupling coefficient is controlled by pulling the waveguide toward the microdisk. The quality factor of the microdisk is measured to be $10^{5}$ thanks to the sidewall smoothing process by hydrogen annealing [175]. The initial gap spacing between the waveguide and the microdisk is $1 \mu \mathrm{m}$. At zero bias, there is literally no coupling, and the microdisk is effectively "turned off." With a voltage applied, the microresonator can switch among undercoupling, critical coupling, or overcoupling regimes dynamically. At critical coupling, the optical transmittance of the through waveguide is suppressed by $30 \mathrm{~dB}$. In the overcoupling regime, the transmission intensity is nearly $100 \%$, while the phases are perturbed around the resonance, similar to the allpass optical filters discussed in the dispersion compensation section. This tunable microdisk resonator has many applications. The group delay and group velocity dispersion can be tuned by varying the gap spacing. A delay time tunable from 27 to 65 ps and dispersion from 185 to $1200 \mathrm{ps} / \mathrm{nm}$ have been experimentally demonstrated [168]. By actuating both input and output waveguides, a reconfigurable optical add/drop multiplexer (ROADM) [176] has also been realized. Multiple tunable microdisks can be integrated to form WSS and WSXC. For telecom applications, high-order resonators are needed to achieve flattop spectral response [170].

Another MEMS microring ROADM was reported by Nielson et al. [169]. They used MEMS actuators to move an optically lossy film to cover the microring. When the film is in contact with the resonator, the quality factor (Q) is significantly lowered, and the resonant wavelength is no longer switched to the drop port. A $60-\mu$ s response time has been measured experimentally. 


\section{B. Photonic Crystals With MEMS Actuators}

Photonic crystals and photonic bandgap materials afford unprecedented control over optical fields. These types of materials and structures are already having an impact on optical MEMS, and because photonic crystal technology is in a very early stage, it is likely that the impact will become more significant in the future. Here, we will point out some of the developments that are very exciting to designers of optical MEMS.

Based on the mechanism of guided resonance [177], photonic crystals can be designed to provide high reflectivity in a single semiconductor film of subwavelength thickness [178][183]. These types of mirrors open up for more compact optical MEMS devices with better temperature characteristics and more robust surfaces than for devices with the metal mirrors used in most optical MEMS applications. High-reflectivity mirrors that do not suffer from the optical field penetration of dielectric stacks also enable compact optical cavities for optical modulators, sources, and sensors.

Photonic crystals can also be dynamically modified by MEMS actuators to create novel optical devices. A variety of different approaches has been proposed and demonstrated. Stretchable photonic crystals [184] allow the complete crystal to be dynamically altered. Photonic crystal waveguide devices have been modulated through evanescent coupling [185], by atomic force microscopy tips [186], and by optical carrier injection [187], and waveguide switches with electrostatic actuation have been demonstrated [188], [189]. Near-field coupling between photonic crystals has been shown to create strong modulation as a function of small relative displacements [190], [191], and the usefulness of this effect has been demonstrated in displacement sensors [192], [193] and optical filters/modulators [194], [195]. The technology is very much in an embryonic stage, and the experimental devices are proof-of-concept demonstrations that are far from ready for commercialization. The field is, however, developing very fast. New and improved application concepts are introduced at a high rate, so, the opportunities for commercial development in the relatively near future seem very promising.

Taking full advantage of these opportunities will require developments in MEMS technology. The very same properties of photonic crystals that make them useful for optical devices also make them extremely sensitive to pattern irregularities and surface defects. Commercial development will therefore require improved MEMS surface treatments and much better lithography than is commonly used for commercial MEMS today.

\section{OTher SWITCHING TeChNOLOGIES}

Although the primary focus of this paper is on MEMS technology, it should be mentioned that several other technologies are also serious contenders for lightwave communications applications. Silica or silicon PLCs provide a guided-wave platform for integrating the switch fabric monolithically. Thermal optically switched PLC has been widely researched. Examples include $16 \times 16$ [196] and $1 \times 128$ [197] matrix switches, $2 \times 2$ WSXC with 16 wavelength channels [150], and $1 \times 9$ WSS with eight channels and $200-\mathrm{GHz}$ spacing
[151]. Switches using microfluidic actuation have also been employed to change TIR conditions in arrays of intersecting waveguides. Examples include Agilent's Champaign switch (32 × 32, also called "bubble" switch) [37] and NTT's OLIVE switches $(16 \times 16)$ [38]. Lithium niobate is attractive for its fast switching speed; however, the chip size tends to be very large and sometimes needs hybrid integration with silica PLC to form large-scale switches [198]. Liquid crystals have been widely used in free-space-based switches and filters because of its electro-optic properties. Recently, liquid crystal-onsilicon has been employed in $1 \times 9$ WSS with programmable bandwidth [199].

\section{CONCLUSION}

We have reviewed recent progresses in optical MEMS for lightwave communication applications. In the past decade, we have witnessed an explosive growth and accelerated maturation of MEMS technologies. Many innovative MEMS devices and optical designs have been introduced. Several components have been transformed from laboratory prototypes into packaged products that meet Telcordia reliability qualifications. Significant progress has been made in VOAs, small $N \times N$ optical switches, medium and large $N \times N$ OXCs, and various wavelength-selective devices such as filters, spectral equalizers and tunable dispersion compensators, WADMs, WSSs and crossconnects, and tunable lasers. In addition to the original purposes, the technologies and expertise developed in the last decade are also available for new emerging applications.

\section{REFERENCES}

[1] K. E. Petersen, "Micromechanical light modulator array fabricated on silicon [laser display applications]," Appl. Phys. Lett., vol. 31, no. 8, pp. 521-523, Oct. 1977.

[2] — , "Silicon torsional scanning mirror," IBM J. Res. Develop., vol. 24, no. 5, pp. 631-637, 1980.

[3] L. J. Hornbeck, "Projection displays and MEMS: Timely convergence for a bright future," presented at the SPIE-Int. Soc. Optical Eng. Conf., vol. 2639, p. 2, 1995.

[4] O. Solgaard, F. S. A. Sandejas, and D. M. Bloom, "Deformable grating optical modulator," Opt. Lett., vol. 17, no. 9, pp. 688-690, May 1992.

[5] K. W. Goossen, J. A. Walker, and S. C. Arney, "Silicon modulator based on mechanically-active antireflection layer with $1 \mathrm{Mbit} / \mathrm{sec}$ capability for fiber-in-the-loop applications," IEEE Photon. Technol. Lett., vol. 6, no. 9, pp. 1119-1121, Sep. 1994.

[6] S. S. Lee, L. Y. Lin, and M. C. Wu, "Surface-micromachined free-space fibre-optic switches," Electron. Lett., vol. 31, no. 17, pp. 1481-1482, Aug. 1995.

[7] M. C. Wu, "Micromachining for optical and optoelectronic systems," Proc. IEEE, vol. 85, no. 11, pp. 1833-1856, Nov. 1997.

[8] R. S. Muller and K. Y. Lau, "Surface-micromachined microoptical elements and systems," Proc. IEEE, vol. 86, no. 8, pp. 1705-1720, Aug. 1998.

[9] L. Y. Lin, E. L. Goldstein, and R. W. Tkach, "Free-space micromachined optical switches for optical networking," IEEE J. Sel. Topics Quantum Electron., vol. 5, no. 1, pp. 4-9, Jan./Feb. 1999.

[10] D. J. Bishop, C. R. Giles, and S. R. Das, "The rise of optical switching," Sci. Amer, vol. 284, no. 1, pp. 88-94, Jan. 2001.

[11] J. E. Ford, "Optical MEMS: Legacy of the telecom boom," in Proc. Solid State Sensor, Actuator and Microsyst. Workshop, Hilton Head, SC, Jun. 6-10, 2004, pp. 1-3.

[12] J. A. Walker, K. W. Goossen, and S. C. Arney, "Fabrication of a mechanical antireflection switch for fiber-to-the-home systems," J. Microelectromech. Syst., vol. 5, no. 1, pp. 45-51, Mar. 1996.

[13] C. Marxer, M. A. Gretillat, V. P. Jaecklin, R. Baettig, O. Anthamatten, and P. Vogel, "Megahertz opto-mechanical modulator," Sens. Actuators A, Phys., vol. 52, no. 1, pp. 46-50, Mar. 1996. 
[14] D. S. Greywall, P. A. Busch, and J. A. Walker, "Phenomenological model for gas-damping of micromechanical structures," Sens. Actuators A, Phys., vol. 72, no. 1, pp. 49-70, Jan. 1999.

[15] C. Marxer, M. A. Gretillat, N. F. de Rooij, R. Battig, O. Anthamatten, B. Valk, and P. Vogel, "Reflective duplexer based on silicon micromechanics for fiber-optic communication," J. Lightw. Technol., vol. 17, no. 1, pp. 115-122, Jan. 1999.

[16] J. E. Ford, J. A. Walker, D. S. Greywall, and K. W. Goossen, "Micromechanical fiber-optic attenuator with 3 microsecond response," J. Lightw. Technol., vol. 16, no. 9, pp. 1663-1670, Sep. 1998.

[17] B. Barber, C. R. Giles, V. Askyuk, R. Ruel, L. Stulz, and D. Bishop, "A fiber connectorized MEMS variable optical attenuator," IEEE Photon. Technol. Lett., vol. 10, no. 9, pp. 1262-1264, Sep. 1998.

[18] C. Marxer, P. Griss, and N. F. de Rooij, "A variable optical attenuator based on silicon micromechanics," IEEE Photon. Technol. Lett., vol. 11, no. 2, pp. 233-235, Feb. 1999.

[19] K. Isamoto, K. Kato, A. Morosawa, C. H. Chong, H. Fujita, and H. Toshiyoshi, "A 5-V operated MEMS variable optical attenuator by SOI bulk micromachining," IEEE J. Sel. Topics Quantum Electron., vol. 10 , no. 3, pp. 570-578, May/Jun. 2004.

[20] A. Godil, "Diffractive MEMS technology offers a new platform for optical networks," Laser Focus World, vol. 38, no. 5, pp. 181-185, 2002.

[21] [Online]. Available: http://www.lightconnect.com/news/news_ release 030205.shtml, 2005

[22] H. Toshiyoshi and H. Fujita, "Electrostatic micro torsion mirrors for an optical switch matrix," J. Microelectromech. Syst., vol. 5, no. 4, pp. 231-237, Dec. 1996.

[23] R. A. Miller, Y. C. Tai, G. Xu, J. Bartha, and F. Lin, "An electromagnetic MEMS $2 \times 2$ fiber optic bypass switch," presented at the Int. Conf. Solid-State Sensors and Actuators, Chicago, IL, 1997, Paper 1A4.

[24] C. Marxer, C. Thio, M. A. Gretillat, N. F. de Rooij, R. Battig, O. Anthamatten, B. Valk, and P. Vogel, "Vertical mirrors fabricated by deep reactive ion etching for fiber-optic switching applications," J. Microelectromech. Syst., vol. 6, no. 3, pp. 277-285, Sep. 1997.

[25] C. Marxer and N. F. de Rooij, "Micro-opto-mechanical $2 \times 2$ switch for single-mode fibers based on plasma-etched silicon mirror and electrostatic actuation," J. Lightw. Technol., vol. 17, no. 1, pp. 2-6, Jan. 1999.

[26] B. Hichwa, M. Duelli, D. Friedrich, C. Iaconis, C. Marxer, M. Mao, and C. Olson, "A unique latching $2 \times 2$ MEMS fiber optics switch," presented at the IEEE Conf. Optical MEMS, Kauai, HI, 2000.

[27] W. Noell, P. A. Clerc, F. Duport, C. Marxer, and N. de Rooij, "Novel process-insensitive latchable $2 \times 2$ optical cross connector for singleand multimode optical MEMS fiber switches," in Proc. IEEE/LEOS Int. Conf. Optical MEMS (Cat. No. 03EX682), Piscataway, NJ, 2003, pp. 49-50.

[28] L. Y. Lin, E. L. Goldstein, and R. W. Tkach, "Free-space micromachined optical switches with submillisecond switching time for large-scale optical crossconnects," IEEE Photon. Technol. Lett., vol. 10, no. 4, pp. 525-527, Apr. 1998.

[29] L. Fan, S. Gloeckner, P. D. Dobblelaere, S. Patra, D. Reiley, C. King, T. Yeh, J. Gritters, S. Gutierrez, Y. Loke, M. Harburn, R. Chen, E. Kruglick, M. Wu, and A. Husain, "Digital MEMS switch for planar photonic crossconnects," in OFC_Postconference Tech. Dig. (IEEE Cat. No. 02CH37339), Washington, DC, 2002, vol. 1, pp. 93-94.

[30] B. Behin, K. Y. Lau, and R. S. Muller, "Magnetically actuated micromirrors for fiber-optic switching," in Proc. Tech. Dig. Solid-State Sensor and Actuator Workshop, Cleveland, OH, 1998, pp. 273-276.

[31] R. L. Wood, R. Mahadevan, and E. Hill, "MEMS 2D matrix switch," in Proc. OFC-Postconference Tech. Dig. (IEEE Cat. No. 02CH37339), Washington, DC, 2002, vol. 1, pp. 91-92.

[32] R. T. Chen, H. Nguyen, and M. C. Wu, "A high-speed low-voltage stressinduced micromachined $2 \times 2$ optical switch," IEEE Photon. Technol. Lett., vol. 11, no. 11, pp. 1396-1398, Nov. 1999.

[33] J.-N. Kuo, G.-B. Lee, and W.-F. Pan, "A high-speed low-voltage doubleswitch optical crossconnect using stress-induced bending micromirrors," IEEE Photon. Technol. Lett., vol. 16, no. 9, p. 2042, Sep. 2004.

[34] L.-Y. Lin, E. L. Goldstein, and R. W. Tkach, "On the expandability of free-space micromachined optical cross connects," J. Lightw. Technol., vol. 18 , no. 4, pp. 482-489, Apr. 2000

[35] M. C. Wu and P. R. Patterson, "Free-space optical MEMS," in MEMS: A Practical Guide to Design, Analysis, and Applications, J. G. Korvink and O. Paul, Eds. Norwich, NY: William Andrew, 2005, pp. 345-402.

[36] P. M. Dobbelaere, S. Gloeckner, S. K. Patra, L. Fan, C. King, and K. Falta, "Design, manufacture and reliability of 2-D MEMS optical switches," in Proc. SPIE-Int. Soc. Opt. Eng., 2003, vol. 4945, pp. 39-45.
[37] J. E. Fouquet, "Compact optical cross-connect switch based on total internal reflection in a fluid-containing planar lightwave circuit," in Proc. Opt. Fiber Commun. Conf. Tech. Dig. Postconference Edition. Trends Opt. and Photon. (IEEE Cat. No. 00CH37079), Washington, DC, 2000, vol. 37, pp. 204-206.

[38] T. Sakata, H. Togo, M. Makihara, F. Shimokawa, and K. Kaneko, "Improvement of switching time in a thermocapillarity optical switch," J. Lightw. Technol., vol. 19, no. 7, pp. 1023-1027, Jul. 2001.

[39] P. M. Hagelin, U. Krishnamoorthy, C. M. Arft, J. P. Heritage, and O. Solgaard, "Scalable fiber optic switch using micromachined mirrors," in Proc. Int. Conf. Solid-State Sens. and Actuators (Transducer), Sendai, Japan, Jun. 7-10, 1999, pp. 782-785.

[40] P. M. Hagelin, U. Krishnamoorthy, J. P. Heritage, and O. Solgaard, "Scalable optical cross-connect switch using micromachined mirrors," IEEE Photon. Technol. Lett., vol. 12, no. 7, pp. 882-884, Jul. 2000.

[41] R. R. A. Syms, "Scaling laws for MEMS mirror-rotation optical cross connect switches," J. Lightw. Technol., vol. 20, no. 7, pp. 1084-1094, Jul. 2002.

[42] D. T. Neilson, R. Frahm, P. Kolodner, C. A. Bolle, R. Ryf, J. Kim, A. R. Papazian, C. J. Nuzman, A. Gasparyan, N. R. Basavanhally, V. A. Aksyuk, and J. V. Gates, "256 × 256 port optical crossconnect subsystem," J. Lightw. Technol., vol. 22, no. 6, pp. 1499-1509, Jun. 2004.

[43] T. Yamamoto, J. Yamaguchi, N. Takeuchi, A. Shimizu, E. Higurashi, R. Sawada, and Y. Uenishi, "A three-dimensional MEMS optical switching module having 100 input and 100 output ports," IEEE Photon. Technol. Lett., vol. 15, no. 10, pp. 1360-1362, Oct. 2003.

[44] M. Yano, F. Yamagishi, and T. Tsuda, "Optical MEMS for photonic switching-compact and stable optical crossconnect switches for simple, fast, and flexible wavelength applications in recent photonic networks," IEEE J. Sel. Topics Quantum Electron., vol. 11, no. 2, pp. 383-394, Mar./Apr. 2005.

[45] X. Zheng, V. Kaman, Y. Shifu, X. Yuanjian, O. Jerphagnon, K. Adrian, R. C. Anderson, H. N. Poulsen, L. Bin, J. R. Sechrist, C. Pusarla, R. Helkey, D. J. Blumenthal, and J. E. Bowers, "Three-dimensional MEMS photonic cross-connect switch design and performance," IEEE J. Sel. Topics Quantum Electron., vol. 9, no. 2, pp. 571-578, Mar./Apr. 2003.

[46] A. Fernandez, B. P. Staker, W. E. Owens, L. P. Muray, J. P. Spallas, and W. C. Banyai, "Modular MEMS design and fabrication for an $80 \times 80$ transparent optical cross-connect switch," Proc. SPIE-Int. Soc. Opt. Eng, vol. 5604, no. 1, pp. 208-217, 2004.

[47] J. Kim, C. J. Nuzman, B. Kumar, D. F. Lieuwen, J. S. Kraus, A. Weiss, C. P. Lichtenwalner, A. R. Papazian, R. E. Frahm, N. R. Basavanhally, D. A. Ramsey, V. A. Aksyuk, F. Pardo, M. E. Simon, V. Lifton, H. B. Chan, M. Haueis, A. Gasparyan, H. R. Shea, S. Arney, C. A. Bolle, P. R. Kolodner, R. Ryf, D. T. Neilson, and J. V. Gates, "1100 × 1100 port MEMS-based optical crossconnect with 4-dB maximum loss," IEEE Photon. Technol. Lett., vol. 15, no. 11, pp. 1537-1539, Nov. 2003.

[48] R. Ryf, J. Kim, J. P. Hickey, A. Gnauck, D. Carr, F. Pardo, C. Bolle, R. Frahm, N. Basavanhally, C. Yoh, D. Ramsey, R. Boie, R. George, J. Kraus, C. Lichtenwalner, R. Papazian, J. Gates, H. R. Shea, A. Gasparyan, V. Muratov, J. E. Griffith, J. A. Prybyla, S. Goyal, C. D. White, M. T. Lin, R. Ruel, C. Nijander, S. Arney, D. T. Neilson, D. J. Bishop, P. Kolodner, S. Pau, C. J. Nuzman, A. Weis, B. Kumar, D. Lieuwen, V. Aksyuk, D. S. Greywall, T. C. Lee, H. T. Soh, W. M. Mansfield, S. Jin, W. Y. Lai, H. A. Huggins, D. L. Barr, R. A. Cirelli, G. R. Bogart, K. Teffeau, R. Vella, H. Mavoori, A. Ramirez, N. A. Ciampa, F. P. Klemens, M. D. Morris, T. Boone, J. Q. Liu, J. M. Rosamilia, and C. R. Giles, "1296-port MEMS transparent optical crossconnect with 2.07 petabit/s switch capacity," in Proc. OFC Tech. Dig. Postconference Edition. Postdeadline Papers (IEEE Cat. No. 01CH37171), Washington, DC, 2001, vol. 4, p. PD28-1-3.

[49] M. Kozhevnikov, N. R. Basavanhally, J. D. Weld, Y. L. Low, P. Kolodner, C. A. Bolle, R. Ryf, A. R. Papazian, A. Olkhovets, F. Pardo, J. Kim, D. T. Neilson, V. A. Aksyuk, and J. V. Gates, "Compact $64 \times 64$ micromechanical optical cross connect," IEEE Photon. Technol. Lett., vol. 15, no. 7, pp. 993-995, Jul. 2003.

[50] V. A. Aksyuk, S. Arney, N. R. Basavanhally, D. J. Bishop, C. A. Bolle, C. C. Chang, R. Frahm, A. Gasparyan, J. V. Gates, R. George, C. R. Giles, J. Kim, P. R. Kolodner, T. M. Lee, D. T. Neilson, C. Nijander, C. J. Nuzman, M. Paczkowski, A. R. Papazian, F. Pardo, D. A. Ramsey, R. Ryf, R. E. Scotti, H. Shea, and M. E. Simon, " $238 \times 238$ micromechanical optical cross connect," IEEE Photon. Technol. Lett., vol. 15, no. 4, p. 587, Apr. 2003.

[51] L. Fan and M. C. Wu, "Two-dimensional optical scanner with large angular rotation realized by self-assembled micro-elevator," in Proc. 
IEEE/LEOS Summer Topical Meeting. Dig. Broadband Opt. Netw. and Technol.: An Emerging Reality. Opt. MEMS. Smart Pixels. Organic Opt. and Optoelectron. (Cat. No. 98TH8369), New York, NY, 1998, pp. $107-108$.

[52] V. A. Aksyuk, F. Pardo, D. Carr, D. Greywall, H. B. Chan, M. E. Simon, A. Gasparyan, H. Shea, V. Lifton, C. Bolle, S. Arney, R. Frahm, M. Paczkowski, M. Haueis, R. Ryf, D. T. Neilson, J. Kim, C. R. Giles, and D. Bishop, "Beam-steering micromirrors for large optical crossconnects," J. Lightw. Technol., vol. 21, no. 3, pp. 634-642, Mar. 2003.

[53] D. S. Greywall, P. A. Busch, F. Pardo, D. W. Carr, G. Bogart, and H. T. Soh, "Crystalline silicon tilting mirrors for optical cross-connect switches," J. Microelectromech. Syst., vol. 12, no. 5, pp. 708-712, Oct. 2003.

[54] R. Sawada, J. Yamaguchi, E. Higurashi, A. Shimizu, T. Yamamoto, N. Takeuchi, and Y. Uenishi, "Single Si crystal 1024 ch MEMS mirror based on terraced electrodes and a high-aspect ratio torsion spring for 3-D cross-connect switch," in Proc. IEEE/LEOS Int. Conf. Optical MEMS (Cat. No. 02EX610), Piscataway, NJ, 2002, pp. 11-12.

[55] N. Kouma, O. Tsuboi, Y. Mizuno, H. Okuda, X. Mi, M. Iwaki, H. Soneda, S. Ueda, and I. Sawaki, "A multi-step DRIE process for a $128 \times 128$ micromirror array," in Proc. IEEE/LEOS Int. Conf. Opt. MEMS (Cat. No. 03EX682), Piscataway, NJ, 2003, pp. 53-54.

[56] T. D. Kudrle, C. C. Wang, M. G. Bancu, J. C. Hsiao, A. Pareek, M. Waelti, G. A. Kirkos, T. Shone, C. D. Fung, and C. H. Mastrangelo, "Single-crystal silicon micromirror array with polysilicon flexures," Sens. Actuators A, Phys., vol. 119, no. 2, pp. 559-566, Apr. 2005.

[57] S. D. Senturia, Microsystem Design. New York: Springer-Verlag, 2004, pp. $130-138$

[58] J. Dadap et al., "Modular MEMS-based optical cross-connect with large port-count optical switch," IEEE Photon. Technol. Lett., vol. 15, no. 12, pp. 1773-1775, Dec. 2003.

[59] R. A. Conant, J. T. Nee, K. Y. Lau, and R. S. Muller, "A flat highfrequency scanning micromirror," in Tech. Dig. Solid-State Sensor and Actuator Workshop (TRF Cat. No. OOTRF-0001), Cleveland, OH, 2000, pp. 6-9.

[60] O. Tsuboi, Y. Mizuno, N. Kouma, H. Soneda, H. Okuda, S. Ueda, I. Sawaki, F. Yamagishi, and Y. Nakamura, "A 2-axis comb-driven micromirror array for 3-D MEMS optical switch," Trans. Inst. Electr. Eng. Jpn., vol. 123-E, no. 10, pp. 398-402, Oct. 2003.

[61] H. Obi, T. Yamanoi, H. Fujita, and H. Toshiyoshi, "A new design for improving stability of electrostatic vertical comb mirrors and its application to fiber optic variable attenuators," Review Laser Eng., vol. 33, pt. 11, pp. 766-771, 2005.

[62] U. Krishnamoorthy, L. Daesung, and O. Solgaard, "Self-aligned vertical electrostatic combdrives for micromirror actuation," J. Microelectromech. Syst., vol. 12, no. 4, pp. 458-464, Aug. 2003.

[63] K.-H. Jeong and L. P. Lee, "A novel microfabrication of a self-aligned vertical comb drive on a single SOI wafer for optical MEMS applications," J. Micromech. Microeng., vol. 15, no. 2, pp. 277-281, Feb. 2005.

[64] W. Piyawattanametha, P. R. Patterson, D. Hah, H. Toshiyoshi, and M. C. Wu, "Surface- and bulk- micromachined two-dimensional scanner driven by angular vertical comb actuators," J. Microelectromech. Syst., vol. 14, no. 6, pp. 1329-1338, Dec. 2005

[65] J. Kim, D. Christensen, and L. Lin, "Monolithic 2-D scanning mirror using self-aligned angular vertical comb drives," IEEE Photon. Technol. Lett., vol. 17, no. 11, pp. 2307-2309, Nov. 2005.

[66] M. Yoda, K. Isamoto, C. Chong, H. Ito, A. Murata, S. Kamisuki, M. Atobe, and H. Toshiyoshi, "A MEMS 1-D optical scanner for laser projection display using self-assembled vertical combs and scan-angle magnifying mechanism," in Proc. TRANSDUCERS-13th Int. Conf. Solid-State Sens., Actuators and Microsyst. Dig. Tech. Papers (IEEE Cat. No. 05TH8791), Piscataway, NJ, 2005, vol. 1, pp. 968-971.

[67] J. E. Ford and J. A. Walker, "Dynamic spectral power equalization using micro-opto-mechanics," IEEE Photon. Technol. Lett., vol. 10, no. 10, pp. $1440-1442$, Oct. 1998

[68] J. E. Ford, K. W. Goossen, J. A. Walker, D. T. Neilson, D. M. Tennant, S. Y. Park, and J. W. Sulhoff, "Interference-based micromechanical spectral equalizers," IEEE J. Sel. Topics Quantum Electron., vol. 10, no. 3, pp. 579-587, May/Jun. 2004.

[69] K. W. Goossen, J. A. Walker, D. T. Neilson, J. E. Ford, and W. H. Knox, "Micromechanical gain slope compensator for spectrally linear optical power equalization," IEEE Photon. Technol. Lett., vol. 12, no. 7, pp. 831-833, Jul. 2000.

[70] C. R. Giles, V. Askyuk, B. Barber, R. Ruel, L. Stulz, and D. Bishop, "A silicon MEMS optical switch attenuator and its use in lightwave subsystems," IEEE J. Sel. Topics Quantum Electron., vol. 5, no. 1, pp. 18-25, Jan./Feb. 1999.
[71] N. A. Riza and M. J. Mughal, "Broadband optical equalizer using fault-tolerant digital micromirrors," Opt. Express, vol. 11, no. 13, pp. 1559-1565, Jun. 2003.

[72] J. I. Trisnadi, C. B. Carlisle, and R. J. Monteverde, "High-performance dynamic gain equalizer for advanced DWDM optical networks," in Proc. SPIE, 2002, vol. 4870, pp. 101-105.

[73] J. J. Bernstein, M. R. Dokmeci, G. Kirkos, A. B. Osenar, J. Peanasky, and A. Pareek, "MEMS tilt-mirror spatial light modulator for a dynamic spectral equalizer," J. Microelectromech. Syst., vol. 13, no. 2, pp. 272-278, Apr. 2004

[74] S. H. Oh and D. M. Marom, "Attenuation mechanism effect on filter shape in channelized dynamic spectral equalizers," Appl. Opt., vol. 43, no. 1, pp. 127-131, Jan. 2004.

[75] D. T. Neilson, R. Ryf, F. Pardo, V. A. Aksyuk, M. E. Simon, D. O. Lopez, D. M. Marom, and S. Chandrasekhar, "MEMS-based channelized dispersion compensator with flat passbands," J. Lightw. Technol., vol. 22, no. 1, pp. 101-105, Jan. 2004.

[76] J. E. Ford, J. A. Walker, V. Aksyuk, and D. J. Bishop, "Wavelengthselectable add/drop with tilting micromirrors," presented at the IEEE Lasers and Electro-Optics Society Annu. Meeting, San Francisco, CA, Nov. 1997, Postdeadline Paper PD2.3.

[77] J. E. Ford, V. A. Aksyuk, D. J. Bishop, and J. A. Walker, "Wavelength add-drop switching using tilting micromirrors," J. Lightw. Technol., vol. 17, no. 5, pp. 904-911, May 1999.

[78] J. K. Rhee, I. Tomkos, and M. J. Li, "A broadcast-and-select OADM optical network with dedicated optical-channel protection," J. Lightw. Technol., vol. 21, no. 1, pp. 25-31, Jan. 2003.

[79] D. T. Neilson, H. Tang, D. S. Greywall, N. R. Basavanhally, L. Ko, D. A. Ramsey, J. D. Weld, Y. L. Low, F. Pardo, D. O. Lopez, P. Busch, J. Prybyla, M. Haueis, C. S. Pai, R. Scotti, and R. Ryf, "Channel equalization and blocking filter utilizing microelectromechanical mirrors," IEEE J. Sel. Topics Quantum Electron., vol. 10, no. 3, pp. 563-569, May/Jun. 2004

[80] D. M. Marom, D. T. Neilson, D. S. Greywall, P. Chien-Shing, N. R Basavanhally, V. A. Aksyuk, D. O. Lopez, F. Pardo, M. E. Simon, Y. Low, P. Kolodner, and C. A. Bolle, "Wavelength-selective $1 \times K$ switches using free-space optics and MEMS micromirrors: Theory, design, and implementation," J. Lightw. Technol., vol. 23, no. 4, pp. 1620-1630, Apr. 2005.

[81] T. Ducellier, J. Bismuth, S. F. Roux, A. Gillet, C. Merchant, M. Miller, M. Mala, Y. Ma, L. Tay, J. Sibille, M. Alavanja, A. Deren, M. Cugalj, D. Ivancevic, V. Dhuler, E. Hill, A. Cowen, B. Shen, and R. Wood "The MWS $1 \times 4$ : A high performance wavelength switching building block," in Proc. ECOC, 2002, pp. 1-2.

[82] J. Tsai, S. Huang, D. Hah, H. Toshiyoshi, and M. C. Wu, "Open-loop operation of MEMS-based $1 \times N$ wavelength-selective switch with longterm stability and repeatability," IEEE Photon. Technol. Lett., vol. 16, no. 4, p. 1041, Apr. 2004.

[83] D. S. Greywall, P. Chien-Shing, O. Sang-Hyun, C. Chorng-Ping, D. M. Marom, P. A. Busch, R. A. Cirelli, J. A. Taylor, F. P. Klemens, T. W. Sorsch, J. E. Bower, W.-C. Lai, and H. T. Soh, "Monolithic fringefield-activated crystalline silicon tilting-mirror devices," J. Microelectromech. Syst., vol. 12, no. 5, pp. 702-707, Oct. 2003.

[84] D. Hah, H. S.-Y. Huang, J.-C. Tsai, J.-C. Toshiyoshi, and M. C. Wu, "Low-voltage, large-scan angle MEMS analog micromirror arrays with hidden vertical comb-drive actuators," J. Microelectromech. Syst., vol. 13, no. 2, pp. 279-289, Apr. 2004

[85] W. P. Taylor, J. D. Brazzle, A. B. Osenar, C. J. Corcoran, I. H. Jafri, D. Keating, G. Kirkos, M. Lockwood, A. Pareek, and J. J. Bernstein, "A high fill factor linear mirror array for a wavelength selective switch," J. Micromech. Microeng., vol. 14, no. 1, pp. 147-152, Jan. 2004.

[86] J. Tsai, H. Sophia Ting-Yu, D. Hah, and M. C. Wu, " $1 \times \mathrm{N}^{2}$ wavelengthselective switch with two cross-scanning one-axis analog micromirror arrays in a 4-f optical system," J. Lightw. Technol., vol. 24, no. 2, pp. 897-903, Feb. 2006

[87] J. C. Tsai and M. C. Wu, "A high port-count wavelength-selective switch using a large scan-angle, high fill-factor, two-axis MEMS scanner array," IEEE Photon. Technol. Lett., vol. 18, no. 13, p. 1439, Jul. 2006.

[88] J.-C. Tsai, L. Fan, C.-H. Chi, D. Hah, and M. C. Wu, "A large port-count $1 \times 32$ wavelength-selective switch using a large scan-angle, high fillfactor, two-axis analog micromirror array," in Proc. 30th ECOC, Kista, Sweden, 2004, vol. 2, pp. 152-153.

[89] J. Tsai and M. C. Wu, "Gimbal-less MEMS two-axis optical scanner array with high fill-factor," J. Microelectromech. Syst., vol. 14, no. 6, p. 1323 , Dec. 2005.

[90] J.-C. Tsai, L. Fan, D. Hah, and M. C. Wu, "A high fill-factor, large scan-angle, two-axis analog micromirror array driven by leverage 
mechanism," presented at the IEEE/LEOS Int. Conf. Optical MEMS and Applications, Takamatsu, Japan, 2004.

[91] V. Kaman, Z. Xuezhe, Y. Shifu, J. Klingshirn, C. Pusarla, R. J. Helkey, O. Jerphagnon, and J. E. Bowers, "A $32 \times 10 \mathrm{~Gb} / \mathrm{s}$ DWDM metropolitan network demonstration using wavelength-selective photonic crossconnects and narrow-band EDFAs," IEEE Photon. Technol. Lett., vol. 17, no. 9, pp. 1977-1979, Sep. 2005.

[92] R. Ryf, P. Bernasconi, P. Kolodner, J. Kim, J. P. Hickey, D. Carr, F. Pardo, C. Bolle, R. Frahm, N. Basavanhally, C. Yoh, D. Ramsey, R. George, J. Kraus, C. Lichtenwalner, R. Papazian, J. Gates, H. R. Shea, A. Gasparyan, V. Muratov, J. E. Griffith, J. A. Prybyla, S. Goyal, C. D. White, M. T. Lin, R. Ruel, C. Nijander, S. Amey, D. T. Neilson, D. J. Bishop, S. Pau, C. Nuzman, A. Weis, B. Kumar, D. Lieuwen, V. Aksyuk, D. S. Greywall, T. C. Lee, H. T. Soh, W. M. Mansfield, S. Jin, W. Y. Lai, H. A. Huggins, D. L. Barr, R. A. Cirelli, G. R. Bogart, K. Teffeau, R. Vella, H. Mavoori, A. Ramirez, N. A. Ciampa, F. P. Klemens, M. D. Morris, T. Boone, J. Q. Liu, J. M. Rosamilia, and C. R. Giies, "Scalable wavelength-selective crossconnect switch based on MEMS and planar waveguides," in Proc. IEEE-PostDeadline Papers. 27th Eur. Conf. Opt. Commun. (Cat. No. 01TH8551), Piscataway, NJ, 2001, vol. 6, pp. 76-77.

[93] A. R. Pratt, B. Charbonnier, P. Harper, D. Nesset, B. K. Nayar, and N. J. Doran, "40/spl times/10.7 Gb/s DWDM transmission over a meshed ULH network with dynamically re-configurable optical cross connects," presented at the Opt. Fiber Commun. Conf. (OFC), Atlanta, GA, 2003, Paper PD9.

[94] D. M. Marom, D. T. Neilson, J. Leuthold, M. A. Gibbons, and C. R. Giles, "64 channel $4 \times 4$ wavelength-selective cross-connect for $40 \mathrm{~Gb} / \mathrm{s}$ channel rates with $10 \mathrm{~Tb} / \mathrm{s}$ throughput capacity," presented at the Eur. Conf. Opt. Commun. (ECOC), Rimini, Italy, Sep. 2003, We4.P.130.

[95] J. H. Jerman and S. R. Mallinson, "A miniature Fabry-Perot interferometer fabricated using silicon micromachining techniques," in Proc. Solid State Sensor and Actuator Workshop Tech. Dig., Hilton Head Island, SC, 1988, pp. 16-18.

[96] A. Tran, Y. H. Lo, Z. H. Zhu, D. Haronian, and E. Mozdy, "Surface micromachined Fabry-Perot tunable filter," IEEE Photon. Technol. Lett., vol. 8, no. 3, pp. 393-395, Mar. 1996.

[97] K. Cao, W. Liu, and J. J. Talghader, "Curvature compensation in micromirrors with high-reflectivity optical coatings," J. Microelectromech. Syst., vol. 10, no. 3, pp. 409-417, Sep. 2001.

[98] E. C. Vail, M. S. Wu, G. S. Li, L. Eng, and C. J. Chang-Hasnain, "GaAs micromachined widely tunable Fabry-Pérot filters," Electron. Lett., vol. 31, no. 3, pp. 228-229, Feb. 1995.

[99] M. C. Larson, B. Pezeshki, and J. S. Harris, "Vertical coupled-cavity microinterferometer on GaAs with deformable-membrane top mirror," IEEE Photon. Technol. Lett., vol. 7, no. 4, pp. 382-384, Apr. 1995.

[100] A. Spisser, R. Ledantec, C. Seassal, J. L. Leclercq, T. Benyattou, D. Rondi, R. Blondeau, G. Guillot, and P. Viktorovitch, "Highly selective and widely tunable $1.55-\mu \mathrm{m}$ InP/air-gap micromachined Fabry-Pérot filter for optical communications," IEEE Photon. Technol. Lett., vol. 10, no. 9, pp. 1259-1261, Sep. 1998.

[101] P. Tayebati, P. Wang, M. Azimi, L. Maflah, and D. Vakhshoori, "Microelectromechanical tunable filter with stable half symmetric cavity," Electron. Lett., vol. 34, no. 20, pp. 1967-1968, Oct. 1998.

[102] M. Garrigues, J. Danglot, J. L. Leclercq, and O. Parillaud, "Tunable highfinesse InP/Air MOEMS filter," IEEE Photon. Technol. Lett., vol. 17, no. 7, pp. 1471-1473, Jul. 2005.

[103] C. J. Chang-Hasnain, "Tunable VCSEL," IEEE J. Sel. Topics Quantum Electron., vol. 6, no. 6, pp. 978-987, Nov./Dec. 2000.

[104] D. Hohlfeld and H. Zappe, "An all-dielectric tunable optical filter based on the thermo-optic effect," J. Opt. A, Pure Appl. Opt., vol. 6, no. 6, pp. 504-511, Jun. 2004.

[105] F. Gires and P. Tournois, "Interferometer utilizable pour la compression d'impulsions lumineuses modules en frequence," C.R. Acad. Sci., vol. 258, no. 5, pp. 6112-6115, 1964.

[106] C. K. Madsen, J. A. Walker, J. E. Ford, K. W. Goossen, T. N. Nielsen, and G. Lenz, "A tunable dispersion compensating MEMS all-pass filter," IEEE Photon. Technol. Lett., vol. 12, no. 6, pp. 651-653, Jun. 2000.

[107] K. Yu and A. Solgaard, "MEMS optical wavelength deinterleaver with continuously variable channel spacing and center wavelength," IEEE Photon. Technol. Lett., vol. 15, no. 3, pp. 425-427, Mar. 2003.

[108] K. Yu and O. Solgaard, "Tunable optical transversal filters based on a Gires-Tournois interferometer with MEMS phase shifters," IEEE J. Sel. Topics Quantum Electron., vol. 10, no. 3, pp. 588-597, May/Jun. 2004.

[109] - "Tunable chromatic dispersion compensators using MEMS Gires-Tournois interferometers," in Proc. IEEE/LEOS Int. Conf. Opt. MEMS, Lugano, Switzerland, 2002, pp. 181-182.
[110] O. Manzardo, H. P. Herzig, C. R. Marxer, and N. F. de Rooij, "Miniaturized time-scanning Fourier transform spectrometer based on silicon technology," Opt. Lett., vol. 24, no. 23, pp. 1705-1707, Dec. 1999.

[111] D. Knipp, H. Stiebig, S. R. Bhalotra, E. Bunte, H. L. Kung, and D. A. B. Miller, "Silicon-based micro-Fourier spectrometer," IEEE Trans. Electron Devices, vol. 52, no. 3, pp. 419-426, Mar. 2005.

[112] S. D. Collins, R. L. Smith, C. Gonzalez, K. P. Stewart, J. G. Hagopian, and J. M. Sirota, "Fourier-transform optical microsystems," Opt. Lett., vol. 24, no. 12, pp. 844-846, Jun. 1999.

[113] K. Yu, D. Lee, U. Krishnamoorthy, N. Park, and O. Solgaard, "Micromachined Fourier transform spectrometer on silicon optical bench platform," Sens. Actuators A, Phys., vol. 130-131, pp. 523-530, 2006.

[114] O. Manzardo, R. Michaely, F. Schadelin, W. Noell, T. Overstolz, N. De Rooij, and H. P. Herzig, "Miniature lamellar grating interferometer based on silicon technology," Opt. Lett., vol. 29, no. 13, pp. 1437-1439, Jul. 2004.

[115] H. L. Kung, S. R. Bhalotra, J. D. Mansell, B. Miller, and J. S. Harris, "Standing-wave transform spectrometer based on integrated MEMS mirror and thin-film photodetector," IEEE J. Sel. Topics Quantum Electron., vol. 8, no. 1, pp. 98-105, Jan./Feb. 2002.

[116] R. A. DeVerse, R. M. Hammaker, and W. G. Fateley, "Realization of the Hadamard multiplex advantage using a programmable optical mask in a dispersive flat-field near-infrared spectrometer," Appl. Spectrosc., vol. 54, no. 12, pp. 1751-1758, Dec. 2000.

[117] J. P. Heritage, A. M. Weiner, and R. N. Thurston, "Picosecond pulse shaping by spectral phase and amplitude manipulation," Opt. Lett., vol. 10, no. 12, pp. 609-611, Dec. 1985.

[118] A. M. Weiner, J. P. Heritage, and J. A. Salehi, "Encoding and decoding of femtosecond pulses," Opt. Lett., vol. 13, no. 4, pp. 300-302, Apr. 1988.

[119] G. J. Tearney, B. E. Bouma, and J. G. Fujimoto, "High-speed phaseand group-delay scanning with a grating-based phase control delay line," Opt. Lett., vol. 22, no. 23, pp. 1811-1813, Dec. 1997.

[120] Y. T. Pan, H. K. Xie, and G. K. Fedder, "Endoscopic optical coherence tomography based on a microelectromechanical mirror," Opt. Lett., vol. 26, no. 24, pp. 1966-1968, Dec. 2001.

[121] K. T. Cornett, P. M. Hagelin, J. P. Heritage, O. Solgaard, and M. Everett, "Miniature variable optical delay using silicon micromachined scanning mirrors," in Proc. CLEO, San Francisco, CA, pp. 383-384.

[122] O. Solgaard, F. S. A. Sandejas, and D. M. Bloom, "Deformable grating optical modulator," Opt. Lett., vol. 17, no. 9, pp. 688-690, May 1992.

[123] R. Apte, F. Sandejas, W. Banyai, and D. Bloom, "Grating light valves for high resolution displays," in Proc. Solid-State Sensors and Actuators Workshop, Hilton Head, SC, Jun. 13-16, 1994, pp. 1-6.

[124] S. Kubota, "The grating light valve projector," Optics Photonics News, vol. 13, no. 9, pp. 50-53, Sep. 2002.

[125] G. G. Yaralioglu, A. Atalar, S. R. Manalis, and C. F. Quate, "Analysis and design of an interdigital cantilever as a displacement sensor," J. Appl. Phys., vol. 83, no. 12, pp. 7405-7415, Jun. 1998.

[126] N. C. Loh, M. A. Schmidt, and S. R. Manalis, "Sub-10 cm(3) interferometric accelerometer with nano-g resolution," J. Microelectromech. Syst., vol. 11, no. 3, pp. 182-187, Jun. 2002.

[127] C. A. Savran, T. P. Burg, J. Fritz, and S. R. Manalis, "Microfabricated mechanical biosensor with inherently differential readout," Appl. Phys. Lett., vol. 83, no. 8, pp. 1659-1661, Aug. 2003.

[128] N. A. Hall and F. L. Degertekin, "Self-calibrating micromachined microphone with integrated optical displacement detection," in Proc. Transducer, Munich, Germany, Jun. 10-14, 2001, pp. 118-121.

[129] T. Perazzo, M. Mao, O. Kwon, A. Majumdar, J. B. Varesi, and P. Norton, "Infrared vision using uncooled microoptomechanical camera," Appl. Phys. Lett., vol. 74, no. 23, pp. 3567-3569, Jun. 1999.

[130] M. B. Sinclair, M. A. Butler, A. J. Ricco, and S. D. Senturia, "Synthetic spectra: A tool for correlation spectroscopy," Appl. Opt., vol. 36, no. 15, pp. 3342-3348, May 1997.

[131] R. Belikov, X. Li, and O. Solgaard, "Programmable optical wavelength filter based on diffraction from a 2 D MEMS micromirror array," in Proc. CLEO, Tech. Dig., Baltimore, MD, Jun. 1-6, 2003.

[132] R. Belikov, C. Antoine-Snowden, and O. Solgaard, "Femtosecond direct space-to-time pulse shaping with MEMS micromirror arrays," in Proc. IEEE/LEOS Int. Conf. Opt. MEMS, Waikoloa, HI, Aug. 18-21, 2003, pp. 24-25.

[133] T. G. Bifano, J. Perreault, R. K. Mali, and M. N. Horenstein, "Microelectromechanical deformable mirrors," IEEE J. Sel. Topics Quantum Electron., vol. 5, no. 1, pp. 83-89, Jan./Feb. 1999.

[134] R. Belikov and O. Solgaard, "Optical wavelength filtering by diffraction from a surface relief," Opt. Lett., vol. 28, no. 6, pp. 447-449, Mar. 2003. 
[135] R. Belikov, "Diffraction-based optical filtering: Theory and implementation with MEMS," Ph.D. dissertation, Stanford Univ., Stanford, CA, 2005.

[136] G. B. Hocker et al., "The polychromator: A programmable MEMS diffraction grating for synthetic spectra," in Proc. Tech. Dig., SolidState Sensor and Actuator Workshop, Hilton Head, SC, Jun. 4-8, 2000, pp. 89-92.

[137] M. A. Butler, E. R. Deutsch, S. D. Senturia, M. B. Sinclair, W. C. Sweatt, D. W. Youngner, and G. B. Hocker, "A MEMS based programmable diffraction grating for optical holography in the spectral domain," in Proc. IEDM, 2001, pp. 909-912.

[138] H. Sagberg, M. Lacolle, I.-R. Johansen, O. Løvhaugen, R. Belikov, O. Solgaard, and A. Sudb $\varnothing$, "Micromechanical gratings for visible and near-infrared spectroscopy," IEEE J. Sel. Topics Quantum Electron., vol. 10, no. 3, pp. 604-613, May/Jun. 2004.

[139] X. Li, C. Antoine, D. Lee, J.-S. Wang, and O. Solgaard, "Tunable blazed gratings," J. Microelectromech. Syst., vol. 13, no. 3, pp. 597-604, Jun. 2006.

[140] C. Antoine, X. Li, J.-S. Wang, and O. Solgaard, "A reconfigurable optical demultiplexer based on a MEMS deformable blazed grating," in Proc. IEEE/LEOS Int. Conf. Opt. MEMS, Jefferson, WI, Aug. 2006, pp. $183-184$

[141] M. G. Littman and H. J. Metcalf, "Spectrally narrow pulsed dye laser without a beam expander," Appl. Opt., vol. 17, no. 14, pp. 2224-2227, Jul. 1978.

[142] W. R. Trutna and L. F. Stokes, "Continuously tuned external-cavity semiconductor-laser," J. Lightw. Technol., vol. 11, no. 8, pp. 1279-1286, Aug. 1993.

[143] R. R. A. Syms and A. Lohmann, "MOEMS tuning element for a Littrow external cavity laser," J. Microelectromech. Syst., vol. 12, no. 6, pp. 921-928, Dec. 2003.

[144] X. M. Zhang, A. Q. Liu, C. Lu, and D. Y. Tang, "Continuous wavelength tuning in micromachined Littrow external-cavity lasers," IEEE J. Quantum Electron., vol. 41, no. 2, pp. 187-197, Feb. 2005.

[145] W. Huang, R. R. A. Syms, J. Stagg, and A. Lohmann, "Precision MEMS flexure mount for a Littman tunable external cavity laser," in Proc. IEE-Sci. Meas. and Technol., 2004, vol. 151, pp. 67-75.

[146] R. Belikov, C. Antoine-Snowden, and O. Solgaard, "Tunable external cavity laser with a stationary deformable MEMS grating," presented at the Conf. Lasers and Electro-Optics (CLEO), San Francisco, CA, May 16-21, 2004, Paper CWL3.

[147] H. Jerman and J. D. Grade, "A mechanically-balanced, DRIE rotary actuator for a high-power tunable laser," in Proc. Solid-State Sensors and Actuators Workshop, Hilton Head, SC, Jun. 2002, pp. 7-10.

[148] D. Anthon, D. King, J. D. Berger, S. Dutta, and A. Tselikov, "Modehop free sweep tuning of a MEMS tuned external cavity semiconductor laser," presented at the Conf. Lasers and Electro-Optics (CLEO), San Francisco, CA, May 16-21, 2004, Paper CWL2

[149] B. Pezeshki, E. Vail, J. Kubicky, G. Yoffe, S. Zou, J. Heanue, P. Epp, S. Rishton, D. Ton, B. Faraji, M. Emanuel, X. Hong, M. Sherback, V. Agrawal, C. Chipman, and T. Razazan, "20-mW widely tunable laser module using DFB array and MEMS selection," IEEE Photon. Technol. Lett., vol. 14, no. 10, pp. 1457-1459, Oct. 2002.

[150] K. Okamoto, M. Okuno, A. Himeno, and Y. Ohmori, "16-channel optical add/drop multiplexer consisting of arrayed-waveguide gratings and double-gate switches," Electron. Lett., vol. 32, no. 16, pp. 1471-1472, Aug. 1996

[151] C. R. Doerr, L. W. Stulz, D. S. Levy, R. Pafchek, M. Cappuzzo, L. Gomez, A. Wong-Foy, E. Chen, E. Laskowski, G. Bogert, and G. Richards, "Wavelength add-drop node using silica waveguide integration," J. Lightw. Technol., vol. 22, no. 12, pp. 2755-2762, Dec. 2004.

[152] D. M. Marom, C. R. Doerr, N. R. Basavanhally, M. Cappuzzo, L. Gomez, E. Chen, A. Wong-Foy, and E. Laskowski, "Wavelength-selective $1 \times 2$ switch utilizing a planar lightwave circuit stack and a MEMS micromirror array," presented at the IEEE/LEOS Optical MEMS, Kagawa, Japan, 2004, Paper C-1.

[153] D. M. Marom, C. R. Doerr, M. Cappuzzo, E. Chen, A. Wong-Foy, and L. Gomez, "Hybrid free-space and planar lightwave circuit wavelengthselective $1 \times 3$ switch with integrated drop-side demultiplexer," in Proc. 31st Eur. Conf. Opt. Commun., Stevenage, U.K., 2005, vol. 4, pp. 993-994.

[154] T. Ducellier, A. Hnatiw, M. Mala, S. Shaw, A. Mank, D. Touahri, D. McMullin, T. Zami, B. Lavigne, P. Peloso, and O. Leclerc, "Novel high performance hybrid waveguide-MEMS $1 \times 9$ wavelength selective switch in a 32-cascade loop experiment," presented at the Eur. Conf. Opt. Commun. (ECOC), Stockholm, Sweden, 2004, Paper Th4.2.2.
[155] D. T. Fuchs, C. R. Doerr, V. A. Aksyuk, M. E. Simon, L. W. Stulz, S. Chandrasekhar, L. L. Buhl, M. Cappuzzo, L. Gomez, A. Wong-Foy, E. Laskowski, E. Chen, and R. Pafchek, "A hybrid MEMS-waveguide wavelength selective cross connect," IEEE Photon. Technol. Lett., vol. 16, no. 1, pp. 99-101, Jan. 2004.

[156] D. M. Marom, C. R. Doerr, M. A. Cappuzzo, C. Evans Yifan, A. Wong-Foy, L. T. Gomez, and S. Chandrasekhar, "Compact colorless tunable dispersion compensator with $1000-\mathrm{ps} / \mathrm{nm}$ tuning range for 40-gb/s data rates," J. Lightw. Technol., vol. 24, no. 1, pp. 237-241, Jan. 2006

[157] M. Kozhevnikov, P. Kolodner, D. T. Neilson, A. R. Papazian, R. E. Frahm, and J. V. Gates, "Integrated array of $1 \times N$ optical switches for wavelength-independent and WDM applications," J. Lightw. Technol., vol. 24, no. 2, pp. 884-890, Feb. 2006.

[158] C.-H. Chi, J. Yao, J.-C. Tsai, M. C. Wu, and K. Okamoto, "Compact $1 \times 8$ MEMS optical switches using planar lightwave circuits," presented at the Opt. Fiber Commun. Conf., (OFC), Los Angeles, CA, Feb. 23-27, 2004, Paper THQ4.

[159] C. H. Chi, J. Tsai, M. C. Lee, D. Hah, and M. C. Wu, "Integrated $1 \times 4$ wavelength-selective switch with on-chip MEMS micromirrors," in Proc. CLEO, 2005, vol. 3, pp. 1732-1743.

[160] B. Jalali, S. Yegnanarayanan, T. Yoon, T. Yoshimoto, I. Rendina, and F. Coppinger, "Advances in silicon-on-insulator optoelectronics," IEEE J. Sel. Topics Quantum Electron., vol. 4, no. 6, p. 938, Nov./Dec. 1998.

[161] C.-H. Chi, J.-C. Tsai, D. Hah, S. Mathai, M. C. M. Lee, and M. C. Wu, "Silicon-based monolithic $4 \times 4$ wavelength-selective cross connect with on-chip micromirrors," in Proc. Opt. Fiber Commun. Conf., Mar. 05-10, 2006

[162] B. E. Little, S. T. Chu, W. Pan, and Y. Kokubun, "Microring resonator arrays for VLSI photonics," IEEE Photon. Technol. Lett., vol. 12, no. 3, pp. 323-325, Mar. 2000.

[163] C. K. Madsen, G. Lent, A. T. Bruce, M. A. Capuzzo, L. T. Gomez, T. N. Nielsen, and I. Brener, "Multistage dispersion compensator using ring resonators," Opt. Lett., vol. 24, no. 22, pp. 1555-1557, Nov. 1999.

[164] X. Qianfan, B. Schmidt, S. Pradhan, and M. Lipson, "Micrometre-scale silicon electro-optic modulator," Nature, vol. 435, no. 7040, pp. 325327, May 2005.

[165] C. Seung June, K. Djordjev, C. Sang Jun, and P. D. Dapkus, "Microdisk lasers vertically coupled to output waveguides," IEEE Photon. Technol. Lett., vol. 15, no. 10, pp. 1330-1332, Oct. 2003.

[166] M. Lipson, "Guiding, modulating, and emitting light on siliconchallenges and opportunities," J. Lightw. Technol., vol. 23, no. 12, pp. 4222-4238, Dec. 2005.

[167] M. C. Lee and M. C. Wu, "MEMS-actuated microdisk resonators with variable power coupling ratios," IEEE Photon. Technol. Lett., vol. 17, no. 5, pp. 1034-1036, May 2005.

[168] M.-C. M. Lee and M. C. Wu, "Tunable coupling regimes of silicon microdisk resonators using MEMS actuators," Opt. Express, vol. 14, no. 11, pp. 4703-4712, May 2006.

[169] G. N. Nielson, D. Seneviratne, F. Lopez-Royo, P. T. Rakich, Y. Avrahami, M. R. Watts, H. A. Haus, H. L. Tuller, and G. Barbastathis, "Integrated wavelength-selective optical MEMS switching using ring resonator filters," IEEE Photon. Technol. Lett., vol. 17, no. 6, pp. 11901192, Jun. 2005.

[170] B. E. Little, S. T. Chu, P. P. Absil, J. V. Hryniewicz, F. G. Johnson, F. Seiferth, D. Gill, V. Van, O. King, and M. Trakalo, "Very highorder microring resonator filters for WDM applications," IEEE Photon. Technol. Lett., vol. 16, no. 10, pp. 2263-2265, Oct. 2004.

[171] D. Geuzebroek, E. Klein, H. Kelderman, N. Baker, and A. Driessen, "Compact wavelength-selective switch for gigabit filtering in access networks," IEEE Photon. Technol. Lett., vol. 17, no. 2, pp. 336-338, Feb. 2005.

[172] T. A. Ibrahim, W. Cao, Y. Kim, J. Li, J. Goldhar, P. T. Ho, and C. H. Lee, "Lightwave switching in semiconductor microring devices by free carrier injection," J. Lightw. Technol., vol. 21, no. 12, pp. 2997-3003, Dec. 2003.

[173] K. Djordjev, C. Seung-June, C. Sang-Jun, and P. D. Dapkus, "Vertically coupled InP microdisk switching devices with electroabsorptive active regions," IEEE Photon. Technol. Lett., vol. 14, no. 8, pp. 1115-1117, Aug. 2002.

[174] _ "Gain trimming of the resonant characteristics in vertically coupled InP microdisk switches," Appl. Phys. Lett., vol. 80, no. 19, pp. 3467-3469, May 2002.

[175] M. C. Lee and M. C. Wu, "Thermal annealing in hydrogen for 3-D profile transformation on silicon-on-insulator and sidewall roughness reduction," J. Microelectromech. Syst., vol. 15, no. 2, pp. 338-343, Apr. 2006 
[176] M.-C. M. Lee and M. C. Wu, "Variable bandwidth of dynamic add-drop filters based on coupling-controlled microdisk resonators," Opt. Lett., vol. 31, no. 16, pp. 2444-2446, 2006.

[177] S. H. Fan and J. D. Joannopoulos, "Analysis of guided resonances in photonic crystal slabs," Phys. Rev. B, Condens. Matter, vol. 65, no. 23, p. 23512 , Jun. 2002.

[178] C. F. R. Mateus, M. C. Y. Huang, Y. F. Deng, A. R. Neureuther, and C. J. Chang-Hasnain, "Ultrabroadband mirror using low-index cladded subwavelength grating," IEEE Photon. Technol. Lett., vol. 16, no. 2, pp. 518-520, Feb. 2004.

[179] O. Kilic, S. Kim, W. Suh, Y. A. Peter, A. S. Sudbo, M. F. Yanik, S. H. Fan, and O. Solgaard, "Photonic crystal slabs demonstrating strong broadband suppression of transmission in the presence of disorders," Opt. Lett., vol. 29, no. 23, pp. 2782-2784, Dec. 2004.

[180] L. Chen, M. C. Y. Huang, C. F. R. Mateus, C. J. Chang-Hasnain, and Y. Suzuki, "Fabrication and design of an integrable subwavelength ultrabroadband dielectric mirror," Appl. Phys. Lett., vol. 88, no. 3, p. 31 102, Jan. 2006.

[181] V. Lousse, W. Suh, O. Kilic, S. Kim, O. Solgaard, and S. H. Fan, "Angular and polarization properties of a photonic crystal slab mirror," Opt. Express, vol. 12, no. 8, pp. 1575-1582, Apr. 2004.

[182] K. B. Crozier, V. Lousse, O. Kilic, S. Kim, S. H. Fan, and O. Solgaard, "Air-bridged photonic crystal slabs at visible and near-infrared wavelengths," Phys. Rev. B, Condens. Matter, vol. 73, no. 11, p. 115 126, Mar. 2006.

[183] E. Bisaillon, D. Tan, B. Faraji, A. G. Kirk, L. Chrowstowski, and D. V. Plant, "High reflectivity air-bridge subwavelength grating reflector and Fabry-Pérot cavity in AlGaAs/GaAs," Opt. Express, vol. 14, no. 7, pp. 2573-2582, Apr. 2006.

[184] W. Park and J. B. Lee, "Mechanically tunable photonic crystal structure," Appl. Phys. Lett., vol. 85, no. 21, pp. 4845-4847, Nov. 2004.

[185] X. Letartre, J. Mouette, J. L. Leclercq, P. R. Romeo, C. Seassal, and P. Viktorovitch, "Switching devices with spatial and spectral resolution combining photonic crystal and MOEMS structures," J. Lightw. Technol., vol. 21, no. 7, pp. 1691-1699, Jul. 2003.

[186] I. Marki, M. Salt, S. Gautsch, U. Staufer, H. P. Herzig, and N. D. Rooij, "Tunable microcavities in two dimensional photonic crystal waveguides," in Proc. IEEE/LEOS Optical MEMs, Oulu, Finland, Aug. 1-4, 2005, pp. 109-110.

[187] I. Marki, M. Salt, H. P. Herzig, R. Stanley, L. El Melhaoui, P. Lyan, and J. M. Fedeli, "Optically tunable microcavity in a planar photonic crystal silicon waveguide buried in oxide," Opt. Lett., vol. 31, no. 4, pp. 513-515, Feb. 2006.

[188] M. C. M. Lee, D. Y. Hah, E. K. Lau, H. Toshiyoshi, and M. Wu, "MEMSactuated photonic crystal switches," IEEE Photon. Technol. Lett., vol. 18, no. 2, pp. 358-360, Jan. 2006.

[189] S. Iwamoto, M. Tokushima, A. Gomyo, H. Yamada, A. Higo, H. Toshiyoshi, H. Fujita, and Y. Arakawa, "Optical switching in photonic crystal waveguide controlled by micro electro mechanical system," in Proc. CLEO/Pacific Rim, Aug. 30-02, 2005, pp. 233-234.

[190] W. Suh, M. F. Yanik, O. Solgaard, and S. H. Fan, "Displacementsensitive photonic crystal structures based on guided resonance in photonic crystal slabs," Appl. Phys. Lett., vol. 82, no. 13, pp. 1999-2001, Mar. 2003.

[191] W. Suh, O. Solgaard, and S. Fan, "Displacement sensing using evanescent tunneling between guided resonances in photonic crystal slabs," J. Appl. Phys., vol. 98, no. 3, p. 33 102, Aug. 2005.

[192] D. W. Carr, J. P. Sullivan, and T. A. Friedmann, "Laterally deformable nanomechanical zeroth-order gratings: Anomalous diffraction studies by rigorous coupled-wave analysis," Opt. Lett., vol. 28, no. 18, pp. 16361638, Sep. 2003.

[193] B. E. N. Keeler, D. W. Carr, J. P. Sullivan, T. A. Friedmann, and J. R. Wendt, "Experimental demonstration of a laterally deformable optical nanoelectromechanical system grating transducer," Opt. Lett., vol. 29, no. 11, pp. 1182-1184, Jun. 2004.

[194] J. Provine, J. Skinner, and D. A. Horsley, "Subwavelength metal grating tunable filter," in Tech. Dig. IEEE Int. Conf. MEMS, Istanbul, Turkey, Jan. 22-26, 2006, pp. 854-857.

[195] S. Nagasawa, T. Onuki, Y. Ohtera, and H. Kuwano, "MEMS tunable optical filter using auto-cloned photonic crystal," in Tech. Dig. 19th IEEE Int. Conf. MEMS, Istanbul, Turkey, Jan. 22-26, 2006, pp. 858-861.

[196] T. Goh, M. Yasu, K. Hattori, A. Himeno, M. Okuno, and Y. Ohmori, "Low loss and high extinction ratio strictly nonblocking $16 \times 16$ thermooptic matrix switch on 6-in wafer using silica-based planar lightwave circuit technology," J. Lightw. Technol., vol. 19, no. 3, pp. 371-379, Mar. 2001.
[197] T. Watanabe, T. Goh, M. Okuno, S. Sohma, T. Shibata, M. Itoh, M. Kobayashi, M. Ishii, A. Sugita, and Y. Hibino, "Silica-based PLC $1 \times 128$ thermo-optic switch," in Proc. 27th Eur. Conf. Opt. Commun. (Cat. No. 01TH8551), Piscataway, NJ, 2001, vol. 2, pp. 134-135.

[198] S. Thaniyavarn, J. Lin, W. Dougherty, T. Traynor, K. Chiu, G. Abbas, M. LaGasse, W. Charczenko, and M. Hamilton, "Compact, low insertion loss $16 \times 16$ optical switch-array modules," in Proc. OFC, 1997, pp. 5-6.

[199] G. Baxter, S. Frisken, D. Abakoumov, Z. Hao, I. Clarke, A. Bartos, and S. Poole, "Highly programmable wavelength selective switch based on liquid crystal on silicon switching elements," in Proc. IEEE OFC/NFOEC, Piscataway, NJ, 2006, p. 3.

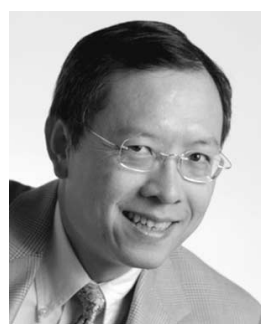

Ming C. Wu (S'82-M'83-SM'00-F'02) received the B.S. degree in electrical engineering from the National Taiwan University, Taipei, Taiwan, R.O.C., and the M.S. and Ph.D. degrees in electrical engineering and computer sciences from the University of California, Berkeley, in 1985 and 1988, respectively.

From 1988 to 1992, he was a member of Technical Staff with AT\&T Bell Laboratories, Murray Hill, NJ. From 1992 to 2004, he was a Professor with the Electrical Engineering Department, University of California, Los Angeles, where he also served as Vice Chair for the Industrial Affiliate Program and as Director of the Nanoelectronics Research Facility. In 2004, he moved to the University of California, Berkeley, where he is currently a Professor of electrical engineering and computer sciences. He is also a Co-Director with the Berkeley Sensor and Actuator Center (BSAC). $\mathrm{He}$ has published six book chapters, over 135 journal papers, and 280 conference papers. He is the holder of 14 U.S. patents. His research interests include optical microelectromechanical systems (MEMS), nanophotonics, biophotonics, and high-speed semiconductor optoelectronics.

Prof. Wu is a member of the Optical Society of America. He was a Packard Foundation Fellow from 1992 to 1997. He was the founding CoChair of IEEE/LEOS Summer Topical Meeting on Optical MEMS (1996): the predecessor of IEEE/LEOS International Conference on Optical MEMS. He has served in the program committees of many conferences, including MEMS, OFC, CLEO, LEOS, MWP, IEDM, DRC, and ISSCC. He was also Guest Editor of two special issues of IEEE journals on Optical MEMS.

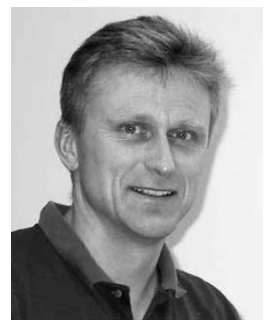

Olav Solgaard (S'88-M'90) received the B.S. degree in electrical engineering from the Norwegian Institute of Technology, Trondheim, Norway, and the M.S. and Ph.D. degrees in electrical engineering from Stanford University, Stanford, CA.

He was a Post-Doctoral Researcher with the University of California, Berkeley, before joining the University of California, Davis, as an Assistant Professor in 1995. In 1999, he joined Stanford University, where he is currently an Associate Professor of electrical engineering. He is a Co-Founder of Silicon Light Machines, Sunnyvale, CA, and an active Consultant in the microelectromechanical systems (MEMS) industry. He has authored more than 170 technical publications and holds 25 patents. His research interests are microoptical and nanooptical devices that combine MEMS, photonic crystals, integrated optics, and free-space optics.

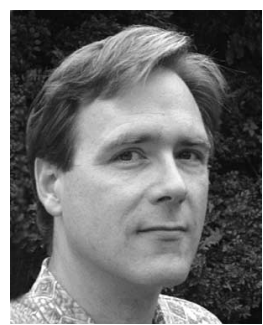

Joseph E. Ford was with the Bell Labs Advanced Photonics Research Department from 1994 to 2000, where he developed microelectromechanical systems (MEMS) and optoelectronic components including the first MEMS variable attenuator, dispersion compensator, spectral equalizer, and wavelength add/drop switch. In 2000, he was with Optical Micro-Machines, becoming Chief Scientist in 2001. In 2002, he joined the University of California, San Diego, where he is currently an Associate Professor of electrical and computer engineering and leads the Photonics Systems Integration Research Lab. He is the coauthor of 45 U.S. patents and over 100 journal articles and conference proceedings.

Dr. Ford was the General Co-Chair of the 2000 IEEE Conference on Optical MEMS and was Program Co-Chair for the 2006 OSA/IEEE Optical Fiber Communications Conference. 